\title{
Second order perturbation of global modes and implications for spanwise wavy actuation
}

\author{
O. Tammisola ${ }^{1} \dagger$, F. Giannetti ${ }^{2}$, V. Citro ${ }^{2}$ and M. P. Juniper ${ }^{1}$ \\ ${ }^{1}$ Dept. of Engineering, University of Cambridge, Trumpington Street, Cambridge CB2 1PZ UK \\ ${ }^{2}$ DIIN, University of Salerno, via Ponte don Melillo, 84084 Fisciano (SA), Italy \\ (Received ?; revised ?; accepted ?. - To be entered by editorial office)
}

Sensitivity analysis has successfully located the most efficient regions in which to apply passive control in many globally unstable flows. As is shown here and in previous studies, the standard sensitivity analysis, which is linear $\left(1^{\text {st }}\right.$ order$)$ with respect to the actuation amplitude, predicts that steady spanwise wavy alternating actuation/modification has no effect on the stability of planar flows, because the eigenvalue change integrates to zero in the spanwise direction. In experiments however, spanwise wavy modification has been shown to stabilize the flow behind a cylinder quite efficiently.

In this paper, we generalize sensitivity analysis by examining the eigenvalue drift (including stabilization/destabilization) up to $2^{\text {nd }}$ order in the perturbation, and show how the $2^{\text {nd }}$ order eigenvalue changes can be computed numerically by overlapping the adjoint eigenfunction with the $1^{\text {st }}$ order global eigenmode correction, shown here for the first time. We confirm the prediction against a direct computation, showing that the eigenvalue drift due to a spanwise wavy base flow modification is of $2^{\text {nd }}$ order. Further analysis reveals that the $2^{\text {nd }}$ order change in the eigenvalue arises through a resonance of the original (2D) eigenmode with other unperturbed eigenmodes that have the same spanwise wavelength as the base flow modification. The eigenvalue drift due to each mode interaction is inversely proportional to the distance between the eigenvalues of the modes (which is similar to resonance), but also depends on mutual overlap of direct and adjoint eigenfunctions (which is similar to pseudoresonance). By this argument, and by calculating the most sensitive regions identified by our analysis, we explain why an in-phase actuation/modification is better than an out-of-phase actuation for control of wake flows by spanwise wavy suction and blowing. We also explain why wavelengths several times longer than the wake thickness are more efficient than short wavelengths.

\section{Key words:}

\section{Introduction}

Control of wake instability by spanwise alternating suction and blowing was studied with DNS by Kim \& Choi (2005). (The alternating suction and blowing will be called "suction" in the rest of the manuscript.) Through steady spanwise wavy suction, they shifted the Hopf bifurcation point of the wake behind a cylinder from $R e \approx 45$ to $R e=140$. This degree of stabilization cannot be achieved with any known 2D open-loop control strategies. Only certain spanwise wavelengths of the actuation were successful at suppressing the instability, however. These wavelengths were approximately $5-6$ cylinder diameters. Control at other wavelengths was less effective. The suction was applied 


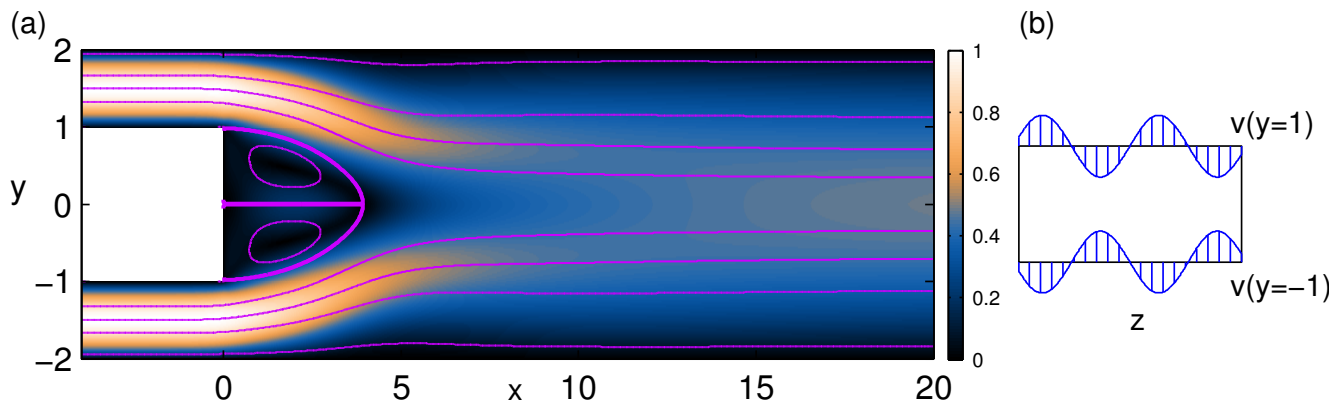

Figure 1. (Color online) (a) Base flow: velocity magnitude and streamlines. The boundary of the recirculation bubble is shown by a thick line. (b) Illustration of in-phase suction.

from two slots placed on the top and the bottom of the cylinder. The configuration in which the suction through the upper slot was in-phase with that through the lower slot (figure $1 \mathrm{~b}$ ) was found to be much more effective than the anti-phase configuration.

In order to explain these effects, Hwang et al. (2013) examined the local absolute instability of a fixed streamwise base flow profile (a wake profile), modified sinusoidally in the spanwise direction. The absolute instability was suppressed by spanwise wavy modifications at medium to long wavelengths, while short wavelengths had a strongly destabilizing effect, the latter not observed in the DNS (Kim \& Choi 2005). The suppression of absolute instability at medium to long wavelengths was proposed to be based on the interaction of von Kármán street with vortex tilting.

Very recently, Del Guercio et al. (2014) examined the effect of streaks in suppressing temporal and absolute instability of wakes. Streamwise streaks were chosen as they are the streamwise uniform perturbations which experience most transient growth around a parallel two-dimensional wake profile taken as a base flow. The optimal initial condition to create the streaks was shown to be streamwise vortices, as in many shear flows. These optimally amplified streaks were then applied as base flow modifications of the local wake profile, and the temporal and absolute growth rates recalculated. Del Guercio et al. (2014) found that the dependence of absolute growth rate on the streak amplitudes was quadratic. Furthermore, they suggested that the spanwise-wavy modifications are particularly effective as stabilisers because they experience strong transient growth in wake flows. This means that high amplitude streaks can be created from low amplitude vortices.

The aim of the present study is to revisit the effect of spanwise wavy steady actuation on stability, using full global analysis of the spatially developing base flow. We explain the findings using a novel generalized sensitivity analysis, which is applicable to spanwise wavy modifications of the base flow and of the linear operator in general. We demonstrate this method on the confined wake behind a flat plate (Fig. 1). Some of the theoretical results are expected to be directly applicable to the cylinder wake configuration. However, this is not our only aim. The framework presented and verified here permits analysis of the effect of spanwise wavy flow perturbations in general (such as streaks), inflow asymmetries in axisymmetric geometries, and control by spanwise wavy actuation.

The $2^{\text {nd }}$ order terms have not been considered in modal stability studies so far (local or global), to the knowledge of the authors. A similar perturbation approach was successfully used in a non-modal study quantifying the effect of streamwise travelling waves on the transient energy growth in a channel flow (Moarref \& Jovanovic 2010; Lieu et al. 2010). 


\section{Perturbation analysis}

Let us consider a general eigensystem of the form:

$$
\mathcal{L} \mathbf{q}_{0}=\sigma_{0} \mathbf{q}_{0},
$$

where $\mathbf{q}_{0}$ is an eigenvector, and $\sigma_{0}$ an eigenvalue. We will now perturb the governing operator by another operator $\delta \mathcal{L}$, so that the problem remains a linear eigenvalue problem. Following a standard perturbation approach (e.g. Hinch (1991),Baumgärtel (1984)), the problem can be expanded in powers of the perturbation amplitude $\epsilon$ :

$(\mathcal{L}+\epsilon \delta \mathcal{L})\left\{\mathbf{q}_{0}+\epsilon \mathbf{q}_{1}+\epsilon^{2} \mathbf{q}_{2}+O\left(\epsilon^{3}\right)\right\}=\left(\sigma_{0}+\epsilon \sigma_{1}+\epsilon^{2} \sigma_{2}+O\left(\epsilon^{3}\right)\right)\left\{\mathbf{q}_{0}+\epsilon \mathbf{q}_{1}+\epsilon^{2} \mathbf{q}_{2}+O\left(\epsilon^{3}\right)\right\}$

Here, $\sigma_{j}$ is the $j^{t h}$ order correction to the eigenvalue, and $\mathbf{q}_{j}(j>0)$ is the $i^{\text {th }}$ order correction to the eigenmode. By grouping the terms at the same order in $\epsilon$, and solving this equation, we will obtain these corrections at different orders.

At the $0^{\text {th }}$ order in $\epsilon$, the original eigenvalue problem is recovered, satisfied by definition. At the $1^{\text {st }}$ order in $\epsilon$, we obtain, after rearrangement:

$$
\left(\mathcal{L}-\sigma_{0} \mathcal{I}\right)\left\{\mathbf{q}_{1}\right\}=-\delta \mathcal{L}\left\{\mathbf{q}_{0}\right\}+\sigma_{1} \mathbf{q}_{0},
$$

where $\mathcal{I}$ is the identity operator. To solve Eq. (2.3), we will use the adjoint eigenmode $\mathbf{q}_{0}^{+}$, defined based on an inner product $\langle$,$\rangle such that \left\langle\mathbf{q}_{0}^{+}, \mathbf{q}_{0}\right\rangle=1$. The adjoint is found by solving the eigenproblem for the adjoint operator: $\mathcal{L}^{+} \mathbf{q}_{0}^{+}=\sigma_{0}^{*} \mathbf{q}_{0}^{+}$(see e.g. Luchini \& Bottaro (2014) for details). The left hand side has no component in the direction of $\mathbf{q}_{0}$, which can be seen by projecting it onto $\mathbf{q}_{0}$ by $\mathbf{q}_{0}^{+}$:

$$
\left\langle\mathbf{q}_{0}^{+},\left(\mathcal{L}-\sigma_{0} \mathcal{I}\right)\left\{\mathbf{q}_{1}\right\}\right\rangle=\left\langle\mathcal{L}^{+}\left\{\mathbf{q}_{0}^{+}\right\}, \mathbf{q}_{1}\right\rangle-\left\langle\mathbf{q}_{0}^{+}, \sigma_{0} \mathbf{q}_{1}\right\rangle=\left\langle\sigma_{0}^{*} \mathbf{q}_{0}^{+}, \mathbf{q}_{1}\right\rangle-\left\langle\mathbf{q}_{0}^{+}, \sigma_{0} \mathbf{q}_{1}\right\rangle=0,
$$

where ${ }^{*}$ denotes the complex conjugate. Hence, the right hand side also must be orthogonal to $\mathbf{q}_{0}^{+}$(Fredholm alternative), giving $0=\left\langle\mathbf{q}_{0}^{+},-\delta \mathcal{L}\left\{\mathbf{q}_{0}\right\}+\sigma_{1} \mathbf{q}_{0}\right\rangle$, which can be rearranged as:

$$
\sigma_{1}=\left\langle\mathbf{q}_{0}^{+}, \delta \mathcal{L}\left\{\mathbf{q}_{0}\right\}\right\rangle .
$$

This $1^{\text {st }}$ order eigenvalue drift is a linear function of the operator perturbation, contains the direct and adjoint eigenmode, and will lead to the standard sensitivity expressions.

At the $2^{\text {nd }}$ order in $\epsilon$, we obtain from Eq. (2.2):

$$
\left(\mathcal{L}-\sigma_{0} \mathcal{I}\right)\left\{\mathbf{q}_{2}\right\}=-\delta \mathcal{L}\left\{\mathbf{q}_{1}\right\}+\sigma_{2} \mathbf{q}_{0}+\sigma_{1} \mathbf{q}_{1} .
$$

By the same argument as for the $1^{\text {st }}$ order, both left and right sides are orthogonal to $\mathbf{q}_{0}$, giving $\sigma_{2}=\left\langle\mathbf{q}_{0}^{+},\left(\delta \mathcal{L}-\sigma_{1} \mathcal{I}\right)\left\{\mathbf{q}_{1}\right\}\right\rangle$. This leads to:

$$
\sigma_{2}=\left\langle\mathbf{q}_{0}^{+}, \delta \mathcal{L}\left\{\mathbf{q}_{1}\right\}\right\rangle .
$$

We observe that an arbitrary component of $\mathbf{q}_{0}$ can always be added to $\mathbf{q}_{1}$ (Hinch 1991), and that Eq. (2.3) would still remain valid. Note that $\sigma_{2}$ remains unaffected by the choice of this component, since $\left\langle\mathbf{q}_{0}^{+}, \delta \mathcal{L}\left\{C \mathbf{q}_{0}\right\}\right\rangle-\sigma_{1}\left\langle\mathbf{q}_{0}^{+}, C \mathbf{q}_{0}\right\rangle=0$ for any constant $C$. The choice of $C$ only corresponds to a normalisation of the total perturbed eigenvector. A convenient choice to guarantee uniqueness and remove the singularity of the left-hand side in Eq. (2.3) is:

$$
\left\langle\mathbf{q}_{0}^{+}, \mathbf{q}_{1}\right\rangle=C=0 .
$$

Note that the $2^{\text {nd }}$ order eigenvalue drift has exactly the same expression as the $1^{\text {st }}$ order drift, but with the eigenmode $\mathbf{q}_{0}$ replaced with the $1^{\text {st }}$ order eigenmode correction $\mathbf{q}_{1}$. This means that all the sensitivity expressions derived in the literature can be used 
straight away to obtain $2^{\text {nd }}$ order corrections, if $\mathbf{q}_{0}$ is replaced by $\mathbf{q}_{1}$. To do this, one of course needs to solve for $\mathbf{q}_{1}$, which can be done numerically as described in section $\S 4$.

\subsection{Application to base flow modifications}

The theory of second order perturbations up to now is not limited to a particular set of equations. Hereafter, we will limit our consideration to perturbations around a steady state (base flow) of the Navier-Stokes equations, leading to an operator perturbation $\delta \mathcal{L}(\delta \mathbf{U})$. A full derivation of the first-order base flow sensitivity can be found in previous works (e.g. Marquet et al. (2008)), and just a brief version is included here in order for the manuscript to be self-contained. The total flow field is governed by the incompressible Navier-Stokes and continuity equations,

$$
\begin{aligned}
\frac{\partial \mathbf{u}_{t o t}}{\partial t}+\mathbf{u}_{t o t} \cdot \nabla \mathbf{u}_{t o t} & =-\nabla p_{t o t}+\frac{1}{R e} \nabla^{2} \mathbf{u}_{t o t} \\
\nabla \cdot \mathbf{u}_{t o t} & =0
\end{aligned}
$$

where $\mathbf{u}_{t o t}=\left(u_{t o t}, v_{t o t}, w_{t o t}\right)$ is a velocity vector, with nondimensional streamwise $\left(u_{t o t}\right)$, vertical $\left(v_{t o t}\right)$ and spanwise $\left(w_{t o t}\right)$ components, and $p_{t o t}$ is the non-dimensional pressure.

In the following, let us denote by capital letters the planar two-dimensional base flow $\mathbf{U}(x, y)=[U(x, y), V(x, y)]$, which is obtained by solving the steady two-dimensional Navier-Stokes equations. Around this base flow we consider linear perturbations, denoted by $\mathbf{u}=[u, v, w]$. Around a planar base flow, a decomposition into bi-global eigenmodes of the following form applies (Theofilis 2003):

$$
\begin{aligned}
\mathbf{u}_{0}(x, y, z, t) & =\left[\hat{u}_{0}(x, y, z), \hat{v}_{0}(x, y, z), \hat{w}_{0}(x, y, z)\right] \exp \left(\sigma_{0} t\right) \\
& =\left[\tilde{u}_{0}(x, y), \tilde{v}_{0}(x, y), \tilde{w}_{0}(x, y)\right] \exp \left(\mathrm{i} \beta_{0} z+\sigma_{0} t\right), \\
p_{0}(x, y, z, t) & =\hat{p}_{0}(x, y, z) \exp \left(\sigma_{0} t\right)=\tilde{p}_{0}(x, y) \exp \left(\mathrm{i} \beta_{0} z+\sigma_{0} t\right),
\end{aligned}
$$

where $\mathbf{u}_{0}=\left(u_{0}, v_{0}, w_{0}\right)$ is a velocity field of the eigenmode, $p_{0}$ is its pressure, $\beta_{0}$ its spanwise wavenumber, and $\sigma_{0}$ its complex temporal eigenfrequency. It should be stressed for later that throughout this manuscript the subscript ^refers to the whole spatial part of the eigenmode including the $z$-dependence, and is used for the two-dimensional part. This distinction is important when taking gradients.

By introducing this ansatz into Navier-Stokes equations, and linearising them, we obtain the bi-global generalized eigenvalue problem:

$$
\begin{aligned}
\sigma_{0} \hat{\mathbf{u}}_{0} & =-\hat{\mathbf{u}}_{0} \cdot \nabla \mathbf{U}_{b}-\mathbf{U}_{b} \cdot \nabla \hat{\mathbf{u}}_{0}-\nabla \hat{p}_{0}+\frac{1}{R e} \nabla^{2} \hat{\mathbf{u}}_{0} \\
\nabla \cdot \hat{\mathbf{u}}_{0} & =0,
\end{aligned}
$$

which we solve with homogeneous Dirichlet boundary conditions for the velocity at all boundaries. The formalism presented for standard eigenvalue problems in the previous section still applies, since it is known that the pressure can be eliminated and hence the generalized eigenvalue problem can be recast into a standard eigenvalue problem. However, the above generalized eigenvalue formulation is convenient to solve numerically.

In what follows, the mode $\left(\mathbf{u}_{0}, p_{0}\right)$ being perturbed will be called the original eigenmode, and its spanwise wavenumber will be denoted by $\beta_{0}$. The spanwise wavenumber of the base flow modification is $\beta_{B}$. The inner product for two velocity vectors $\hat{\mathbf{u}}_{a}$ and 
$\hat{\mathbf{u}}_{b}$ is here defined as:

$$
\begin{aligned}
\left\langle\hat{\mathbf{u}}_{a}, \hat{\mathbf{u}}_{b}\right\rangle & =\lim _{L_{Z} \rightarrow \infty} L_{Z}^{-1} \int_{-L_{Z} / 2}^{L_{Z} / 2} \int_{D(x, y)} \hat{\mathbf{u}}_{a}^{* T} \hat{\mathbf{u}}_{b} \quad d x d y d z \\
& =\lim _{L_{Z} \rightarrow \infty} L_{Z}^{-1} \int_{-L_{Z} / 2}^{L_{Z} / 2} \int_{D(x, y)}\left(\hat{u}_{a}^{*} \hat{u}_{b}+\hat{v}_{a}^{*} \hat{v}_{b}+\hat{w}_{a}^{*} \hat{w}_{b}\right) \quad d x d y d z
\end{aligned}
$$

where $D(x, y)$ is the $2 \mathrm{D}$ flow domain, and $T$ denotes the transpose. The adjoint linearised Navier-Stokes operator is defined using the generalized Lagrange identity based on this inner product, with homogeneous boundary conditions for the perturbation, and the adjoint system becomes:

$$
\begin{aligned}
\sigma_{0}^{+} \hat{\mathbf{u}}_{0}^{+} & =\left(\nabla \mathbf{U}_{b}\right)^{T} \cdot \hat{\mathbf{u}}_{0}^{+}-\mathbf{U}_{b} \cdot \nabla \hat{\mathbf{u}}_{0}^{+}-\nabla \hat{p}_{0}^{+}-\frac{1}{R e} \nabla^{2} \hat{\mathbf{u}}_{0}^{+} \\
\nabla \cdot \hat{\mathbf{u}}_{0}^{+} & =0
\end{aligned}
$$

where it can be shown that $\sigma_{0}^{+}=-\sigma_{0}^{*}$, while $T$ denotes a transpose of the 9 -component gradient matrix (in tensor notation: $\left[\nabla \mathbf{U}_{b}^{T}\right]_{i j}=\partial U_{b, j} / \partial x_{i}$ ). The adjoint eigenproblem also has homogeneous Dirichlet boundary conditions.

By imposing a base flow perturbation $\delta \mathbf{U}$ onto Eq. (??) we obtain:

$$
\sigma_{1}=\left\langle\mathbf{q}_{0}^{+}, \delta \mathcal{L}(\delta \mathbf{U}) \mathbf{q}_{0}\right\rangle=\left\langle\hat{\mathbf{u}}_{0}^{+},-\hat{\mathbf{u}}_{0} \cdot \nabla \delta \mathbf{U}_{b}-\delta \mathbf{U}_{b} \cdot \nabla \hat{\mathbf{u}}_{0}\right\rangle
$$

By integration by parts, and with the homogeneous boundary conditions, the $1^{\text {st }}$ order drift is found to be:

$$
\sigma_{1}=\left\langle\mathbf{q}_{0}^{+}, \delta \mathcal{L}(\delta \mathbf{U}) \mathbf{q}_{0}\right\rangle=\left\langle-\nabla \hat{\mathbf{u}}_{0}^{T *} \hat{\mathbf{u}}_{0}^{+}+\nabla \hat{\mathbf{u}}_{0}^{+} \hat{\mathbf{u}}_{0}^{*}, \delta \mathbf{U}\right\rangle .
$$

Here, the expression $-\nabla \hat{\mathbf{u}}_{0}^{T *} \hat{\mathbf{u}}_{0}^{+}+\nabla \hat{\mathbf{u}}_{0}^{+} \hat{\mathbf{u}}_{0}^{*}$ is precisely the $\left(1^{\text {st }}\right.$-order $)$ sensitivity to base flow modifications (Marquet et al. 2008).

The $2^{\text {nd }}$ order eigenvalue change with respect to base flow modifications is correspondingly:

$$
\sigma_{2}=\left\langle\mathbf{q}_{0}^{+}, \delta \mathcal{L}(\delta \mathbf{U}) \mathbf{q}_{1}\right\rangle=\left\langle-\nabla \hat{\mathbf{u}}_{1}^{T *} \hat{\mathbf{u}}_{0}^{+}+\nabla \hat{\mathbf{u}}_{0}^{+} \hat{\mathbf{u}}_{1}^{*}, \delta \mathbf{U}\right\rangle
$$

where $\hat{\mathbf{u}}_{1}$ is the $1^{\text {st }}$ order correction to the velocity eigenmode, which can be obtained from:

$$
\begin{aligned}
-\hat{\mathbf{u}}_{1} \cdot \nabla \mathbf{U}_{b}-\mathbf{U}_{b} \cdot \nabla \hat{\mathbf{u}}_{1}-\nabla \hat{p}+\frac{1}{R e} \nabla^{2} \hat{\mathbf{u}}_{1}-\sigma \hat{\mathbf{u}}_{1} & =\hat{\mathbf{u}}_{0} \cdot \nabla \delta \mathbf{U}_{b}+\delta \mathbf{U}_{b} \cdot \nabla \hat{\mathbf{u}}_{0} \\
\nabla \cdot \mathbf{u}_{1} & =0 .
\end{aligned}
$$

\subsection{Wavy base flow modifications}

The $1^{\text {st }}$ order eigenvalue correction vanishes for spanwise wavy modifications of $2 \mathrm{D}$ base flows, because the $1^{\text {st }}$ order eigenvalue correction is linear with respect to the base flow modification, and hence the total contribution from a $z$-alternating base flow modification integrates to zero in the $z$-direction. To see this, we consider example base flow modifications of the form $\delta \mathbf{U}=\delta \tilde{\mathbf{U}}(x, y) \cos \left(\beta_{B} z\right)=\delta \tilde{\mathbf{U}}(x, y)\left[\exp \left(\mathrm{i} \beta_{B} z\right)+\exp \left(-\mathrm{i} \beta_{B} z\right)\right] / 2$. Using Eq. (2.13) and (2.15) we obtain:

$\sigma_{1}=\left(\lim _{L_{Z} \rightarrow \infty} L_{Z}^{-1} \int_{-L_{Z} / 2}^{L_{Z} / 2} \cos \left(\beta_{B} z\right) d z\right) \int_{D(x, y)}\left(-\nabla \hat{\mathbf{u}}_{0}^{T} \hat{\mathbf{u}}_{0}^{+*}+\nabla \hat{\mathbf{u}}_{0}^{+*} \hat{\mathbf{u}}_{0}\right) \cdot \delta \hat{\mathbf{U}}(x, y) d x d y=0$,

because the $z$-integral vanishes. 
The $2^{\text {nd }}$ order correction $\sigma_{2}$ remains finite, however, because the $1^{\text {st }}$ order eigenmode correction is of the form: $\mathbf{u}_{1}=\hat{\mathbf{u}}_{1+}(x, y) \exp \left(\mathrm{i}\left(\beta+\beta_{B}\right) z\right)+\hat{\mathbf{u}}_{1-}(x, y) \exp \left(\mathrm{i}\left(\beta-\beta_{B}\right) z\right)$. The reason for this form is that Eq. (2.17) is linear, and the wavenumber is uniquely set by the eigenmode and base flow modification through the right hand side terms. After some algebra we obtain (please note the inclusion of spanwise gradients):

$$
\sigma_{2}=1 / 2 \int_{D(x, y)}\left(-\nabla \hat{\mathbf{u}}_{1}^{T} \hat{\mathbf{u}}_{0}^{+*}+\nabla \hat{\mathbf{u}}_{0}^{+*} \hat{\mathbf{u}}_{1}\right) \cdot \delta \tilde{\mathbf{U}}(x, y) d x d y .
$$

\section{Physical conclusions from the perturbation theory}

One can conclude from $\S 2$ that the $2^{\text {nd }}$ order eigenvalue correction is proportional to the $1^{\text {st }}$ order eigenmode correction. Hence, qualitatively, base flow modifications that induce a large eigenmode correction will induce a large $2^{\text {nd }}$ order eigenvalue drift.

The eigenmode correction is given by (2.17). The left hand side operator is reminiscent of a resolvent operator. As for a resolvent, there are two ways to obtain a large eigenmode correction: (1) The base flow modification is such that $\hat{\mathbf{u}}_{1}$ is close to an eigenmode of the system (which is similar to modal resonance), or (2) the base flow modification invokes a large non-modal response (which is similar to pseudoresonance). These properties become obvious when expanding $\hat{\mathbf{u}}_{1}$ in the basis of the original eigenmodes following (Hinch 1991): $\mathbf{u}_{1}=\sum_{N \neq 0}\left[\sigma_{0}-\sigma_{(N)}\right]^{-1}\left\langle\hat{\mathbf{u}}_{(N)}^{+}, \delta \mathcal{L} \hat{\mathbf{u}}_{0}\right\rangle \hat{\mathbf{u}}_{(N)}+C \hat{\mathbf{u}}_{0}$, where $N$ represents a mode index in the original eigenmode basis, and $C$ is an arbitrary constant. Observe that again $\hat{\mathbf{u}}_{(N)}$ refers to the spatial part of the Navier-Stokes eigenmode number $N$, excluding its time-varying part. It is easily verified that this solution satisfies (2.3), and leads to the following $2^{\text {nd }}$ order eigenvalue correction:

$$
\sigma_{2}=\sum_{N \neq 0}\left[\sigma_{0}-\sigma_{(N)}\right]^{-1}\left\langle\hat{\mathbf{u}}_{(N)}^{+}, \delta \mathcal{L} \hat{\mathbf{u}}_{0}\right\rangle\left\langle\hat{\mathbf{u}}_{0}^{+}, \delta \mathcal{L} \hat{\mathbf{u}}_{(N)}\right\rangle .
$$

Two restrictions need to be mentioned relating to this expansion. Firstly, the expansion does not provide physical results in the limit $\epsilon\left[\sigma_{0}-\sigma_{(N)}\right]^{(-1)} \approx O(1)$, being in fact singular when $\sigma_{(N)} \rightarrow \sigma_{0}$.

The singularity occurs because when $\sigma_{(N)} \rightarrow \sigma_{0}$, then $\left.\left\langle\hat{\mathbf{u}}_{(N)}^{+}, \delta \mathcal{L} \hat{\mathbf{u}}_{0}\right\rangle\left\langle\hat{\mathbf{u}}_{0}^{+}, \delta \mathcal{L} \hat{\mathbf{u}}_{(N)}\right\rangle\right\rangle \rightarrow$ $\left\langle\hat{\mathbf{u}}_{0}^{+}, \delta \mathcal{L} \hat{\mathbf{u}}_{0}\right\rangle^{2}$, which is finite, whereas $\left[\sigma_{0}-\sigma_{(N)}\right]^{-1} \rightarrow \infty$, and therefore $\sigma_{2} \rightarrow \infty$. In fact, it can be shown that if higher order corrections are formed, the sum of all eigenvalue corrections will diverge when $\epsilon\left[\sigma_{0}-\sigma_{(N)}\right]^{(-1)} \gtrsim O(1)$. A similar situation occurs for example when predicting orbits in a three-planet system (Kolmogorov 1954), yielding a similar expansion with respect to distance. If the orbits of two planets are too close, the perturbation approach becomes invalid and other approaches such as the KAM theory need to be applied. In our case the restriction has the following meaning: the expansion as well as the perturbation approach itself are valid as long as the perturbation amplitude is not too large to satisfy $\epsilon\left[\sigma_{0}-\sigma_{(N)}\right]^{(-1)}<O(1)$. If two eigenvalues are very close, the perturbation approach is valid only for small perturbation amplitudes.

In the special case of spanwise wavy base flow modifications, this means that when $\beta_{B}$ is very close to zero, the sensitivities become arbitrarily large; however, the expansion itself is valid for smaller and smaller amplitudes, and hence conclusions derived from it of less practical interest in that regime. The reason for the singularity lies in the structure of the eigenmode spectrum for different $\beta$. When $\beta$ is varied, the most unstable eigenvalue is a smooth continuous function of $\beta$. Only eigenmodes with wavelength $\beta=\beta_{0} \pm \beta_{B}$ contribute to the sum (3.1). When $\beta_{B} \rightarrow 0$, then $\sigma \rightarrow \sigma_{0}$ for the most unstable mode 
with $\beta=\beta_{0} \pm \beta_{B}$. The following can be taken as a practical guideline: when the base flow modification amplitude $\epsilon$ has been chosen, the theory can be applied for $\beta_{B}$ large enough (spanwise wavelength small enough) so that:

$$
\left|\sigma_{0}-\sigma_{(N)}\left(\beta_{B}\right)\right|>\epsilon \quad \forall N .
$$

For $\epsilon=0.01$ in $\S 5.3$ of the manuscript, this translates to the following lower limit for spanwise wavenumber: $\beta_{B}>0.15$, whereas for $\epsilon=0.1$, the upper limit would be approximately $\beta_{B}>0.4$.

Secondly, the eigenvalue that is perturbed needs to belong to the discrete spectrum for the chosen $\beta_{0}$, and a rigorous treatment of the continuous branches in the above sum would require a decomposition of them into a finite number of discrete "leaky modes" (Pralits et al. 2007).

Despite the two restrictions, the above expansion gives significant insights into the physics of general $2^{\text {nd }}$ order perturbations, and wavy perturbations in particular as detailed in $\S 5.2$. Base flow perturbations invoke at the $2^{\text {nd }}$ order a linear resonance between different eigenmodes, similar to the linear frequency response. The eigenvalue drift induced by another eigenmode depends on how close the eigenvalue is to the one being perturbed (modal resonance), and mutual products between adjoint and direct eigenmodes (non-modal effect / pseudoresonance). The two integrands could be large in two different regions of space, and could be affected by two different physical mechanisms, which creates possibilities for rich dynamics. In other words, a non-local base flow modification is not a simple integral of many local base flow modifications. This property is not a consequence of approximations but an inherent physical property of wavy actuation or modification (due to their second order nature) .

\section{Numerical solution}

The numerical results discussed in the present study are carried out using two different codes: the finite element software FreeFEM++ (see http://www.freefem.org) and the Spectral Element Solver (SEM) Nek5000. Three different problems are addressed from a numerical point of view: base flow computations (FreeFem++ and Nek5000), eigenmode computations (FreeFem ++ and Nek5000), and the computation of the first order eigenvector correction $($ FreeFem ++$)$.

In FreeFem ++, the finite-element method is used to solve the base flow, eigenmode and eigenmode correction equations in $\S 2$. The spatial domain is discretized by a triangular finite elements mesh using a Delaunay-Voronoi algorithm, leading to a mesh with 213620 triangles and 108486 vertices. We employ the pair $P 2-P 1$, consisting of piece-wise quadratic velocities and piece-wise linear pressure (Taylor-Hood elements), leading to $10^{6}$ degrees of freedom. After derivation of the variational formulation of the governing equations $(\S 2)$, the associated sparse matrices are built by means of the FreeFem ++ software.

In FreeFem++, the steady version of Eq. (2.7) is solved by the Newton-Raphson iterative procedure to compute the steady base flow for the stability analyses. Second, the direct and adjoint eigenproblems (Eq. 2.12 and 2.14) are solved using the Implicitly Restarted Arnoldi method implemented in the ARPACK library (Lehoucq et al. 1998). Third, the numerical solution of the eigenvector correction $\mathbf{q}_{1}$ is performed, as detailed in a separate section below. All the required matrix inversions are performed using the sparse LU solver UMFPACK (Davis 2004). The iterative procedure is stopped when the $L_{2}$ norm of the residual (the latest correction of the Newton iterations) of the governing equations is less than $10^{-7}$. 


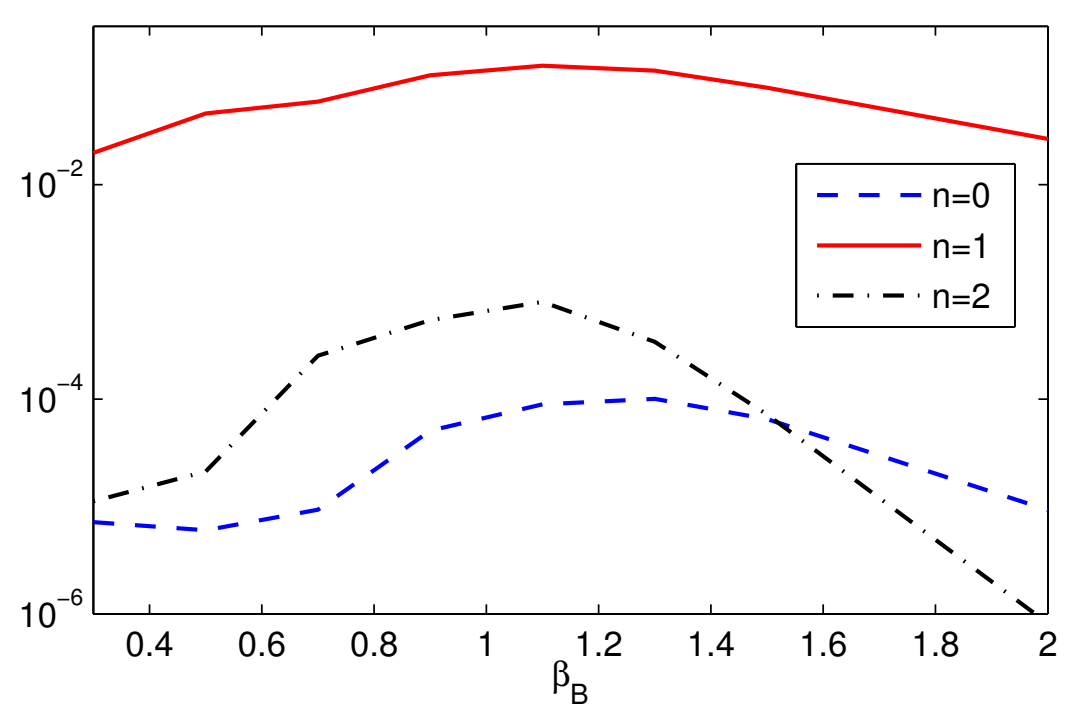

Figure 2. (Color online) The $L_{2}$-norm of the first three spatial coefficients $\delta \mathbf{U}_{n}$ of the Fourier transform of the base flow difference $\delta \mathbf{U}$, at different suction wavelengths $\beta_{B}$. This shows that the $n=1$ - component $\left(\propto \exp \left(\mathrm{i} \beta_{B} z\right)\right)$ dominates. The $y$-scale is logarithmic.

The code chosen for the computation of 3D (suction-modified) base flows and for the TriGlobal linear stability analysis used for method verification purposes is Nek5000 (Fischer 1997). TriGlobal stability means that the eigenmodes are computed directly from the ansatz $\mathbf{u}(x, y, z, t)=\hat{\mathbf{u}}(x, y, z) \exp (\sigma t)$, without setting a spanwise wavenumber. For these verification cases, since the aim is to compare stability characteristics, also the unmodified 2D base flow is computed in Nek5000 and subsequently interpolated into FreeFem ++ . The spatial discretisation for the TriGlobal stability problem (the verification points in figures 3 and 8, and the eigenmode correction verification in figure 6 d-f) consists of 43200 spectral elements, with a gradually refined element distribution in the streamwise direction around the downstream edge of the flat plate, and a finer distribution near the walls in the vertical direction. The spanwise direction is discretised by 12 uniformly distributed elements.

Nek5000 is chosen because it allows a highly accurate matrix-free solution of the TriGlobal stability problems in the present work. As for the finite element method, the incompressible Navier-Stokes equations are cast into a weak form and integrated over the computational domain. A classical Galerkin approximation is used to spatial discretize the governing equations where each velocity field is related to polynomials two degrees higher than the pressure $\left(\mathbb{P}_{N^{-}} \mathbb{P}_{N-2}\right.$ formulation). In this work, the polynomial order $p=5$ was chosen for $3 \mathrm{D}$ computations, and $p=8$ for the $2 \mathrm{D}$ base flow. The space associated with the velocity is spanned by $N$ th-order Lagrange interpolants $h_{i}^{N}$, based on a tensor-product grid formed by Gauss-Lobatto-Legendre nodes in each coordinate direction.

The base flows are computed by time-stepping the nonlinear Navier-Stokes equations on the same grid as for the stability problem, but using only the upper half of the flow domain. At the centreline, we set a symmetry condition, which eliminates the (antisymmetric) oscillatory modes, and the flow converges towards a steady state. The convergence is considered to be reached when two successive velocity fields, separated by 10 non dimensional time units, have a maximum absolute difference of $10^{-6}$. 
The full three-dimensional eigenpairs of the Linearized Navier-Stokes operator are computed (as in Lashgari et al. 2013) using the linearized DNS time-stepper (available in NEK5000) coupled with the Implicit Restart Arnoldi Method implemented in PARPACK (Maschhoff \& Sorensen 1996). Finally, for the 3D cases, the base flows and base flow modifications (see $\S 4.1$ ) are extracted from Nek5000 and interpolated into FreeFem ++ as follows. To simplify the computational procedure, we assume the base flow modification to be linear with respect to the suction amplitude, and hence have the same wavenumber $\beta_{B}$. If the base flow modification is nonlinear (as it might be for high suction amplitudes), then the base flow modification might contain harmonics at multiples of $\beta_{B}$. Then, a rigorous treatment of these requires extracting the harmonics, and computing eigenvector corrections for each harmonic separately. In figure 2 , we have extracted the $L_{2}$-norms of the amplitudes of $\exp \left(\mathrm{i} n \beta_{B} z\right), n=0,1,2$, at different $\beta_{B}$. This shows that the components for $n \neq 1$ are smaller by two orders of magnitude compared to $n=1$, and hence, it is a good approximation to assume that the base flow difference is of the form $\delta \mathbf{U}(x, y) \exp \left(\mathrm{i} \beta_{B} z\right)$. With this assumption, the base flow difference takes the following form:

$$
\delta \mathbf{U}=\mathbf{F}(x, y) \cos \left(\beta_{B} z\right)+\mathbf{G}(x, y) \sin \left(\beta_{B} z\right)
$$

where $\mathbf{F}=\delta \mathbf{U}(z=0)$ and $\mathbf{G}=\delta \mathbf{U}\left(z=\pi /\left[2 \beta_{B}\right]\right)$.

Hence, we interpolate the Nek5000 base flow velocities at $z=0$ and $z=\pi /\left(2 \beta_{B}\right)$ into FreeFem ++ and use the above form for the base flow difference. The interpolation proceeds as follows: First, all the $p=5$ base flows are interpolated in Nek5000 to the Gauss-Lobatto-Legendre points of order $p=8$ (used for the 2D base flow). Second, a triangulation is built in FreeFem ++ around these points. Third, the base flows are interpolated to the final finite element grid inside FreeFem ++ .

\subsection{Second order perturbation}

Since the solution of the $2^{\text {nd }}$ order perturbation problem is new, it is worth mentioning the different ways to solve it numerically. There are two ways to obtain $\sigma_{2}$ for a known operator perturbation (e.g base flow change). The first is to solve equation (2.17) directly for $\mathbf{u}_{1}$, and then compute $\sigma_{2}$ from Eq. (2.5). The second is to use the eigenmode expansion (3.1) by solving another $2 \mathrm{D}$ global eigenvalue problem, and there is no general rule as to how many modes are needed for the sum to converge.

The choice between the two methods depends on what kind of information is requested. If the exact form of the desired base flow change is known (as in $\S 5.3$, where the base flow change by suction was computed explicitly), then computing the eigenvector correction is clearly preferable. If the exact form of the base flow change is unknown, forming the first few terms in the expansion may provide the desired physical insights. In fact, the first term in the sum is a more computationally efficient way to approximate the sensitivity core than traversing a Dirac delta function over the $x$-y-plane, which will be done in $\S 5.2$.

The left-hand side of Eq. (2.3) and its specific form Eq. (2.17), are singular as discussed in $\S 2$, which does not affect $\sigma_{2}$ but may place a requirement on the solver. To prevent singularity, we wish to impose the condition $\left\langle\hat{\mathbf{u}}_{0}^{+}, \hat{\mathbf{u}}_{1}\right\rangle=0$. This is true whenever $\hat{\mathbf{u}}_{1}$ has a spanwise wavenumber different from $\beta_{0}$. As mentioned in $\oint 2.2$, the correct way is to assume that $\mathbf{u}_{1}$ must have the same wavelength as $-\delta \mathcal{L}\left\{\mathbf{u}_{0}\right\}: \beta_{1}=\beta_{0} \pm \beta_{B}$. By replacing $\partial / \partial z$ with $i \beta_{1}$ in the equations, we hence automatically impose the above condition, and $\left(\mathcal{L}\left(\beta_{1}\right)-\sigma_{o} \mathcal{I}\right)$ becomes non-singular. 
The equation system derived from (2.3) becomes:

$$
\begin{array}{r}
-\hat{\mathbf{u}}_{(1,+)} \cdot \nabla \mathbf{U}_{b}-\mathbf{U}_{b} \cdot \nabla \hat{\mathbf{u}}_{(1,+)}-\nabla \hat{p}_{1,+}+\frac{1}{R e} \nabla^{2} \hat{\mathbf{u}}_{(1,+)}-\sigma \hat{\mathbf{u}}_{(1,+)}= \\
\hat{\mathbf{u}}_{(1,+)} \cdot \nabla \delta \mathbf{U}_{+}+\delta \mathbf{U}_{+} \cdot \nabla \hat{\mathbf{u}}_{(1,+)} \\
\nabla \cdot \hat{\mathbf{u}}_{(1,+)}= \\
0 \\
-\hat{\mathbf{u}}_{(1,-)} \cdot \nabla \mathbf{U}_{b}-\mathbf{U}_{b} \cdot \nabla \hat{\mathbf{u}}_{(1,-)}-\nabla \hat{p}_{(1,-)}+\frac{1}{R e} \nabla^{2} \hat{\mathbf{u}}_{(1,-)}-\sigma \hat{\mathbf{u}}_{(1,-)}= \\
\hat{\mathbf{u}}_{(1,-)} \cdot \nabla \delta \mathbf{U}_{-}+\delta \mathbf{U}_{-} \cdot \nabla \hat{\mathbf{u}}_{(1,-)} \\
\nabla \cdot \hat{\mathbf{u}}_{(1,-)}=
\end{array}
$$

where $\partial\left(\delta \mathbf{U}_{ \pm}\right) / \partial z= \pm i \beta_{B} \mathbf{U}$, and $\partial \hat{\mathbf{u}}_{1, \pm} / \partial z=i\left(\beta_{0} \pm \beta_{B}\right) \mathbf{U}$. The base flow change components are found by decomposing the base flow difference as follows:

$$
\delta \mathbf{U}=\delta \mathbf{U}_{+} \exp \left(\mathrm{i} \beta_{B} z\right)+\delta \mathbf{U}_{-} \exp \left(-\mathrm{i} \beta_{B} z\right),
$$

where $\delta \mathbf{U}_{+}$and $\delta \mathbf{U}_{-}$are obtained from Eq. (4.1) in a complex form. The above system is solved by the same Newton-Raphson algorithm as the base flow, but the algorithm converges at one iteration because the system is linear. Finally, the second order perturbation of the eigenvalue was extracted by taking the following integral in FreeFem++:

$$
\sigma_{2}=\left\langle-\hat{\mathbf{u}}_{0}^{+}, \hat{\mathbf{u}}_{(1,+)} \cdot \nabla \delta \mathbf{U}_{-}+\delta \mathbf{U}_{-} \cdot \nabla \hat{\mathbf{u}}_{(1,+)}+\hat{\mathbf{u}}_{(1,-)} \cdot \nabla \delta \mathbf{U}_{+}+\delta \mathbf{U}_{+} \cdot \nabla \hat{\mathbf{u}}_{(1,-)}\right\rangle
$$

\section{Results}

To demonstrate the second order perturbation method, we investigate as an example case the wake behind a flat plate with half thickness $h$, confined in a channel of height $4 h$. The inflow velocity is parabolic on both sides of the plate. The problem is nondimensionalized with $h$, and maximum inflow velocity $U_{i n}$. The Reynolds number is fixed at $R e=100$ for all results presented here. The numerical domain extends from $x=-10$ to $x=60$ in the streamwise direction, and we employ a no-slip condition at the plate and the channel walls.

\subsection{Verification of quadratic behaviour}

Firstly, we need to show that the eigenvalue drift indeed increases quadratically when the amplitude of the base flow modification is increased, that is, the eigenvalue sensitivity is of a $2^{\text {nd }}$ order. Secondly, we want to compare our predicted eigenvalue drifts to the actual eigenvalue drifts when the base flow is modified and the eigenvalues recomputed.

For the purpose of this test, we have chosen the same artificially modified 1D base flow profile as Hwang et al. (2013), multiplied by a Gaussian in the streamwise direction:

$$
\delta U=A\left[\exp \left(-\left(x^{2}+1 / 2(y-1)^{2}\right)\right)+\exp \left(-\left(x^{2}+1 / 2(y+1)^{2}\right)\right)\right] \cos (z)
$$

This base flow modification results in a co-flow at and around the trailing edge of the plate. Note that this test base flow does not satisfy the Navier-Stokes equations, which is necessary for the sake of method validation. It is impossible to introduce base flow modifications which have exactly the same shape but at different amplitudes, so that the total flow satisfies Navier-Stokes. The procedure is comparable to the addition of optimal streaks to the local base flow profile in Del Guercio et al. (2014), with the same shape, but at different amplitudes. 

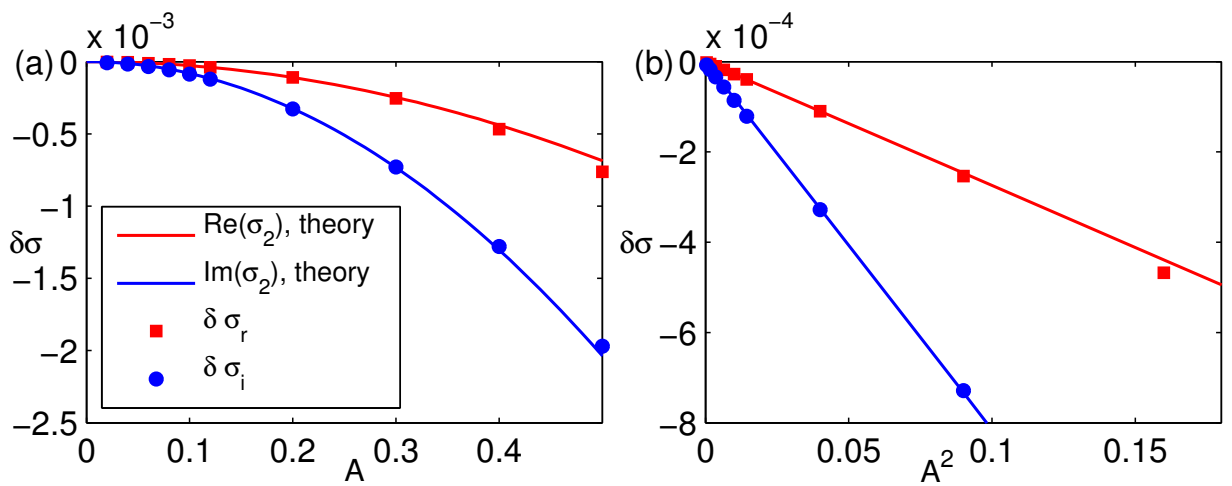

Figure 3. (Color online) (a) Markers: The change of the global mode eigenvalue $\delta \sigma=\delta \sigma_{r}+\mathrm{i} \delta \sigma_{i}$, as a function of the amplitude $A$ of a given base flow modification (Eq. 5.1). Solid lines: The predicted eigenvalue perturbation at the second order $\left(\sigma_{2}\right)$, real and imaginary part. (b) The same as (a), but as a function of $A^{2}$, demonstrating that $\delta \sigma \propto A^{2}$.

The amplitude of the base flow modification is varied from $A=0.01$ (1\% of the maximum inlet velocity) to $A=0.5$ ( $50 \%$ of the maximum inlet velocity). There is no reason to expect a quantitative agreement at the highest amplitudes even from a $2^{\text {nd }}$ order prediction. The results are shown in Fig. 3. The computed changes in both frequency and growth rate are clearly quadratic with respect to the amplitude. The quantitive agreement between prediction and computation is almost exact for $A<0.4$, and as expected deviates more for larger amplitudes. Nevertheless, especially the frequency component agrees very well even for the largest amplitudes.

\subsection{Sensitivity core for spanwise wavy base flow changes}

Next, we show how the theory can be used not only to predict, but also to physically understand, the influence of spanwise wavy perturbations. In the following, we aim to generalise the concept of wavemaker or core of the instability for first-order sensitivities (Giannetti \& Luchini 2007; Marquet et al. 2008), and compare this region for wavy and non-wavy modifications. To do this, we quantify the regions where localized modifications of the base flow similar to a Dirac delta function produce the largest eigenvalue drift. The analogy for first-order sensitivities should not be pushed too far, however. Considering the eigenvalue drift as a sum over eigenmodes as in Eq. (3.1), we see that the eigenvalue drifts for large and non-local changes are not obtained from a single integral over many small changes, but rather from a product of two integrals. For a specific global base flow modification, these two integrals could potentially be large in two different regions. Hence, unlike first-order sensitivity base flow modifications, for the second order perturbations one cannot conclusively obtain all information about the effect of non-local base flow changes from a spatial map obtained for the Dirac delta function.

However, by applying the Dirac delta function we can identify regions where the local flow response for control with each spanwise wavelength is maximum, and hence which are always likely to provide a large contribution. To identify such regions in the flow, to be called "the sensitivity core", we consider the following highly localised base flow modification:

$\delta U=B \cos \left(\beta_{B} z\right)\left[\exp \left(-500\left(\left(x-x_{0}\right)^{2}+\left(y-y_{0}\right)^{2}\right)\right)+\exp \left(-500\left(\left(x-x_{0}\right)^{2}+\left(y+y_{0}\right)^{2}\right)\right)\right]$,

$\delta V=\delta W=0$ 

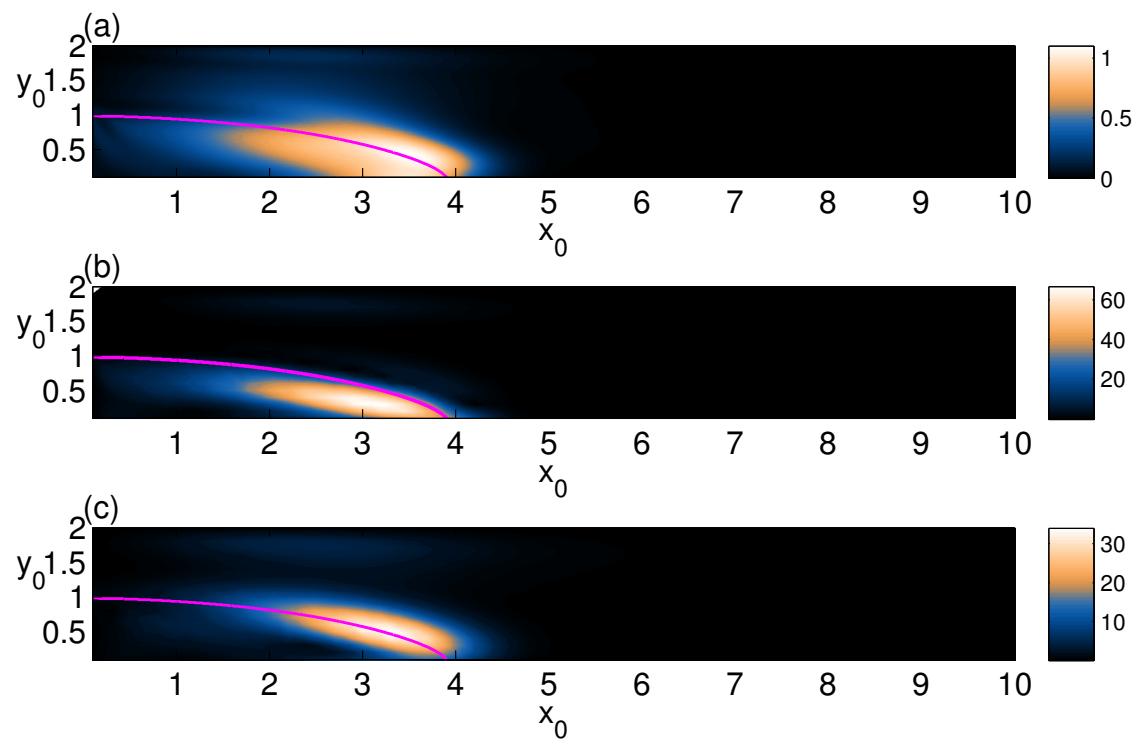

Figure 4. (Color online) The sensitivity core for spanwise wavy base flow modifications estimated by a spatial mapping of the eigenvalue drifts induced by localized modifications approximating Dirac delta function: (a) $\beta_{B}=0$ (2D), (b) $\beta_{B}=1$, (c) $\beta_{B}=30$. The eigenvalue drift is large inside the light-coloured region. This demonstrates that when the same base flow modification is applied at different spanwise wavelengths, long wavelengths are more efficient than short (see colorbars) and much more efficient than 2D suction, while the sensitivity cores have similar shapes as in $2 \mathrm{D}$. The boundary of the recirculation zone is depicted by the solid line (magenta online).

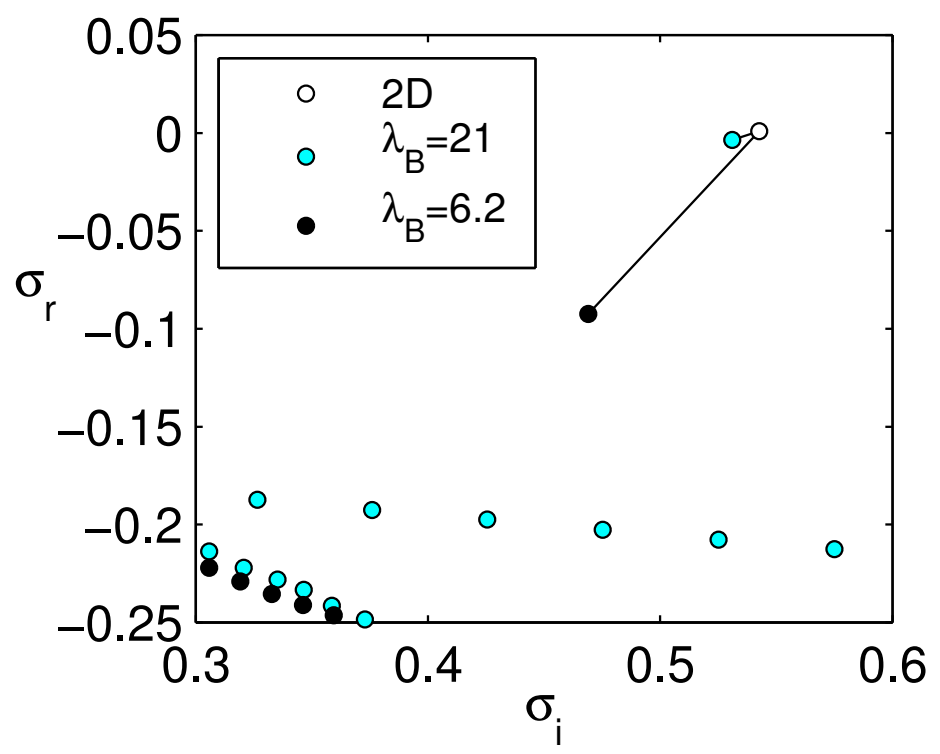

FiguRE 5. (Color online) Unperturbed eigenvalue spectra for two different spanwise wavelengths (see legend), shown together with the unstable $2 \mathrm{D}$ eigenvalue $(\beta=0)$. The solid lines are added to illustrate the distances between the most unstable eigenvalue and the $2 \mathrm{D}$ eigenvalue (white circle). The mode resonance effect ( $\S 3$ ) is inversely proportional to the distance between eigenvalues. 
The base flow change is then normalised to a unit area in the $x-y$-plane by setting the amplitude as:

$$
B=\left[\int \delta \tilde{U}(x, y) d x d y\right]^{-1}
$$

This procedure approximates the effect of a Dirac delta function, but avoids discontinuities which could potentially cause numerical problems.

This base flow modification is traversed over the $x$ - $y$-plane, and the eigenvalue drift is computed for each $\left(x_{0}, y_{0}\right)$-pair. Only the streamwise velocity is shown here, but we tried a Gaussian modification of vertical and spanwise velocity in a few positions too, and these tests indicated that the most efficient regions were similar. The resulting sensitivity core is shown in Fig. 4. Frame (a) shows the standard 2D sensitivity, frame (b) shows the sensitivity core for $\beta_{B}=1$, and frame (c) shows $\beta_{B}=30$. This leads to two interesting observations. Firstly, the longer spanwise wavelength $\left(\beta_{B}=1\right)$ causes a larger eigenvalue change than the $2 \mathrm{D}$ modification by a factor of 60 (see colorbars), and a larger eigenvalue change than the short spanwise wavelength $\left(\beta_{B}=30\right)$ by a factor of 2 . Secondly, all three sensitivities are localized along the recirculation bubble, and have their maximum at $x \approx 3-3.5$.

A physical explanation for both phenomena - the efficiency of long wavelengths and the similar positions of maximum sensitivity for wavy and non-wavy modifications - is found from the eigenmode expansion (3.1). For spanwise wavy perturbations of wavenumber $\beta_{B}$ around a 2D flow, the base flow sensitivity arises through the mode interactions in the sum (3.1). These only involve modes with $\beta= \pm \beta_{B}$. No other modes contribute. For a wake behind a flat plate, the most unstable eigenvalue varies continuously as $\beta$ varies. The eigenvalue spectra at two different values of $\beta$ are shown in Fig. 5, together with the $2 \mathrm{D}(\beta=0)$ eigenvalue $\sigma_{0}$ that is being perturbed. At small $\beta$ (long wavelengths), such as $\beta=0.3$ in Fig. 5 , the leading eigenvalue is very close to the $2 \mathrm{D}$ eigenvalue $(\beta=0)$, while the other eigenmodes are comparatively far away. Therefore, at small wavenumbers, the interaction between the most unstable mode at $\beta= \pm \beta_{B}$ and the original mode at $\beta=0$ provides a large contribution. Qualitatively, the smaller the wavenumber is, the larger the effect on the eigenvalue is (keeping the restrictions of $\S 3$ in mind). Furthermore, the most unstable mode does not change its spatial shape much between $\beta=0$ and small nonzero $\beta$. This means that the sensitivity core remains similar for any small spanwise wavenumbers. At slightly larger wavenumbers (such as $\beta_{B}=1$ in Fig. 5), corresponding to shorter wavelengths, the leading modes are further apart, which means that the effect on $\sigma_{0}$ from the mode interactions is smaller. In this case, other eigenmodes also contribute to the sum, and therefore to the spatial sensitivity distribution, in a similar proportion as the leading mode. However, the wake or shear layer modes which contribute most to the sum have similar frequencies to the wake mode, and these are not essentially different in their spatial distribution, which still gives rise to similar sensitivity regions, but at slightly lower amplitudes.

\subsection{Control effect of steady spanwise wavy suction}

Finally, we apply the theory to study and explain the effect of actual base flow modifications induced by suction on a wake behind a flat plate. A sinusoidal steady wall-normal suction is applied from a slot located near the trailing edge $-0.2<x<-0.01$, similarly to Kim \& Choi (2005): $\mathbf{U}_{\text {wall }}=\left(0,0.01 \cos \left(\beta_{B} z\right), 0\right)$. The suction distributions on the upper and lower surfaces of the plate are in-phase with each other (see Fig. $1 \mathrm{~b}$ for illustration). 
The choice of an in-phase suction distribution (rather than out-of-phase) is worth mentioning, since this choice turns out to be well grounded in the present theory. In the earlier works (Kim \& Choi 2005; Hwang et al. 2013), in-phase distribution was observed to be much more efficient in stabilizing the flow than out-of-phase distribution, but this effect was not explained. The $\sigma_{2}$ derived in the present work (Eq. 3.1) contains an integral product of the mode, its adjoint and the base flow change over $y$. In order for this integral not to vanish, in-phase perturbations may only invoke a resonance of the original mode with sinuous modes, while anti-phase perturbations invoke a resonance with varicose modes. The global eigenvalues for varicose modes in wakes are very damped, so the distance between the original eigenvalue and varicose eigenvalues is long. On the other hand, the eigenvalue distance between the original mode and the leading sinuous mode is short, if the spanwise wavelength for suction $\lambda_{B}=2 \pi / \beta_{B}$ is long, by the arguments in the previous section. Therefore the theory shows that long wavelength in-phase base flow modifications induce the largest eigenvalue drift through the resonance of the original mode with the leading spanwise wavy sinuous mode with wavenumber $\pm \beta_{B}$.

This in-phase suction is implemented as a boundary condition in the base flow computations for 12 different wavenumbers:

$\beta_{B}=(0(2 D), 0.3,0.5,0.7,0.9,1.1,1.3,1.5,1.7,1.9,2,30)$. The difference between the base flows with and without suction is then extracted, as described in $\S 4$. From this point, we proceed in two ways: (1) by computing the eigenvalue change $\sigma_{2}$ based on our theory, and (2) by recomputing the stability around the 3D modified base flow, to get a reference result. For the computation of $\sigma_{2}, \delta \hat{\mathbf{U}}(x, y)$ is extracted from the 3D base flow as described in $\S 4$. The $2 \mathrm{D}$ non-wavy base flow modification is normalized to have the same $L_{2}$-norm as the spanwise wavy ones, to ensure a similar suction effort.

The first order eigenvector correction $\hat{\mathbf{u}}_{1}$ is computed in the process, and this quantity is shown for the first time for linear global modes in figure $6(\mathrm{a}-\mathrm{c})$. Both real and imaginary parts are computed, and $\hat{\mathbf{u}}_{1}$ is observed to have the following form: $\mathbf{u}_{1}=\tilde{\mathbf{u}}_{1}(x, y) \cos \left(\beta_{B} z\right), \mathbf{v}_{1}=\tilde{\mathbf{u}}_{1}(x, y) \cos \left(\beta_{B} z\right), \mathbf{w}_{1}=\tilde{\mathbf{w}}_{1}(x, y) \sin \left(\beta_{B} z\right)$, following the same symmetry in $z$ as the suction, and the induced base flow modification. The different velocity components are shown in figure $6(\mathrm{a}-\mathrm{c})$, for $\beta_{B}=1.1$, and these are reminiscent of eigenfunctions of confined wakes as expected. To further validate our method of computing the eigenvector correction, a true eigenmode correction extracted from a computed 3D eigenmode is shown in figure $6(\mathrm{~d}-\mathrm{f})$. The latter result was obtained from a $3 \mathrm{D}$ eigenmode with suction, $\mathbf{u}_{s}$, by subtracting the component in the direction of the original eigenmode: $\mathbf{u}_{s}-\left\langle\mathbf{u}_{0}^{+}, \mathbf{u}_{s}\right\rangle$. The result is nearly indistuingishable from the one computed from our theory. Both corrections are normalised to a unit $L_{2}$-norm.

The norm of $\mathbf{u}_{1}$ which will be obtained in an actual computation of experiment is arbitrary. The reason is worth discussing in some depth. The norm of $\mathbf{u}_{1}$ is uniquely determined by the norm of the unperturbed (original) eigenvector $\mathbf{u}_{0}$. Conversely, when $\mathbf{u}_{1}$ is known, so that $\left\langle\mathbf{u}_{0}^{+}, \mathbf{u}_{1}\right\rangle=0$, then $\mathbf{u}_{0}$ can be determined uniquely by inverting Eq. (2.3). However, as mentioned in $\S 2$, an arbitrary component $C \mathbf{u}_{0}$ can always be added to $\mathbf{u}_{1}$ to obtain (non-unique) solutions to (2.3). The actual eigenmode returned from a $3 \mathrm{D}$ computation or an experiment may therefore have any value of $C$. The value of $C$ can be found from $\mathbf{u}_{1}$, by inverting (2.3), if desired. Importantly, the eigenvalue correction $\sigma_{2}$ remains independent of $C$ as shown in $\S 2$.

The eigenmode correction has its largest amplitude at $x \approx 5$. However, the relative amplitude of the correction and the original eigenmode determined the final shape, and this depends on the (arbitrary) value of $C$. Hence, the eigenmode correction observed in computation or experiments might be either small or large, while the observed eigenvalue 
(a)

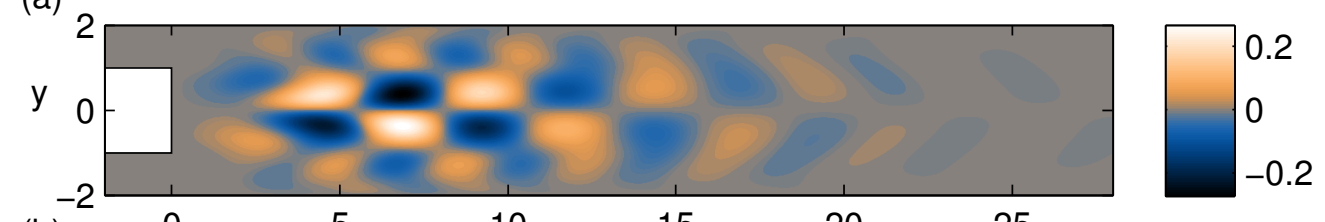

(b)
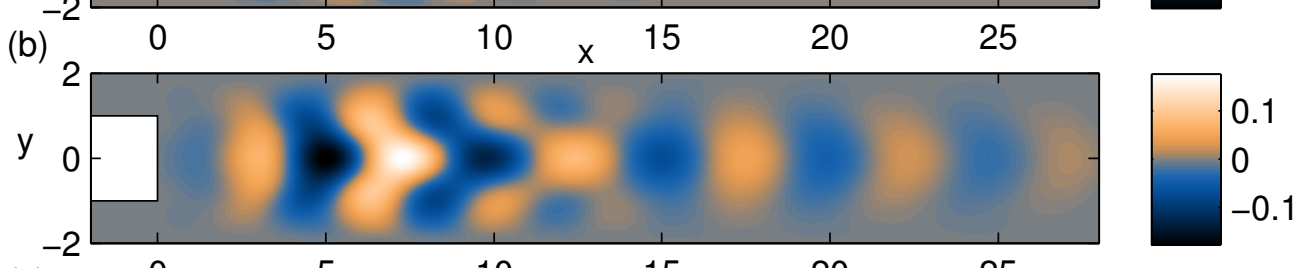

(c)
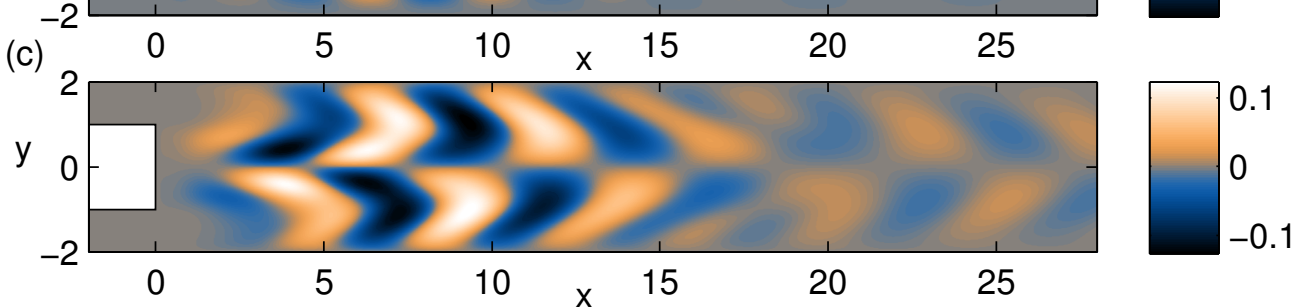

(d)

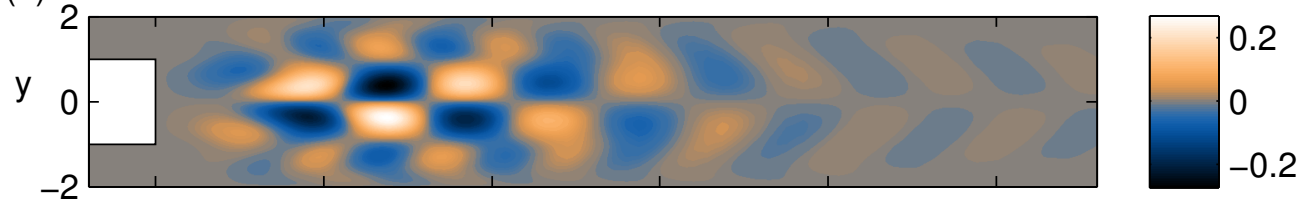

(e)

$-2$

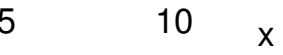

15

20

25
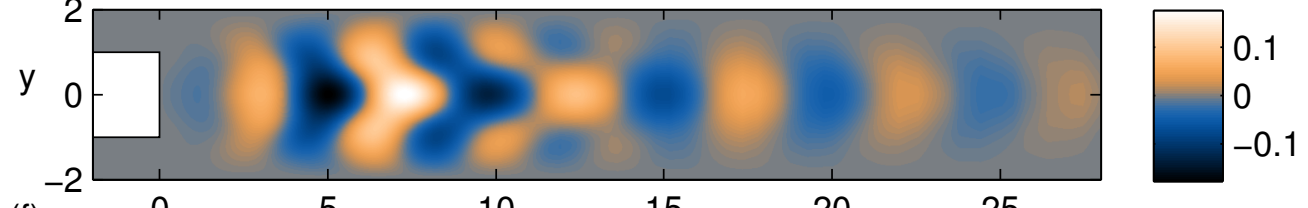

(f)

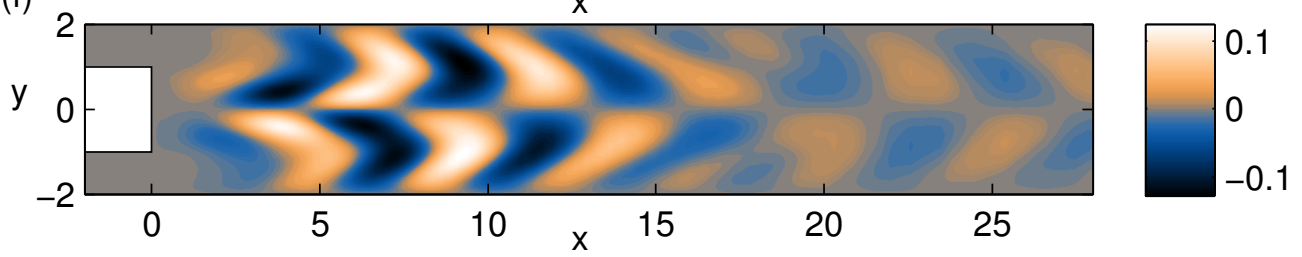

Figure 6. (Color online) (a)-(c): The first order eigenfunction correction computed from Eq. (2.3) with suction wavelength $\beta_{B}=1.1, \hat{\mathbf{u}}_{1}=\left[\tilde{u}_{1}(x, y) \cos \left(\beta_{B} z\right), \tilde{v}_{1}(x, y) \cos \left(\beta_{B} z\right), \tilde{w}_{1}(x, y) \sin \left(\beta_{B} z\right)\right]$, (a) $\tilde{u}_{1}$, real part (b) $\tilde{v}_{1}$, real part, and (c) $\tilde{w}_{1}$, real part. (d)-(f): The same as (a)-(c), but where a true eigenfunction correction is extracted from a computed $3 \mathrm{D}$ eigenfunction with suction $\mathbf{u}_{s}$ by subtracting the component in the direction of the unperturbed eigenfunction. Both correction fields are normalised to a unit $L_{2}$-norm.

correction is unique. Figure 7 shows a computed TriGlobal eigenmode with suction. In the shape of the eigenmode, the spanwise variation only shows further downstream (around $x=8$ ), where the amplitude of the original $2 \mathrm{D}$ eigenmode is small.

When it comes to changing the eigenvalue, however, neither the shape of the correction 


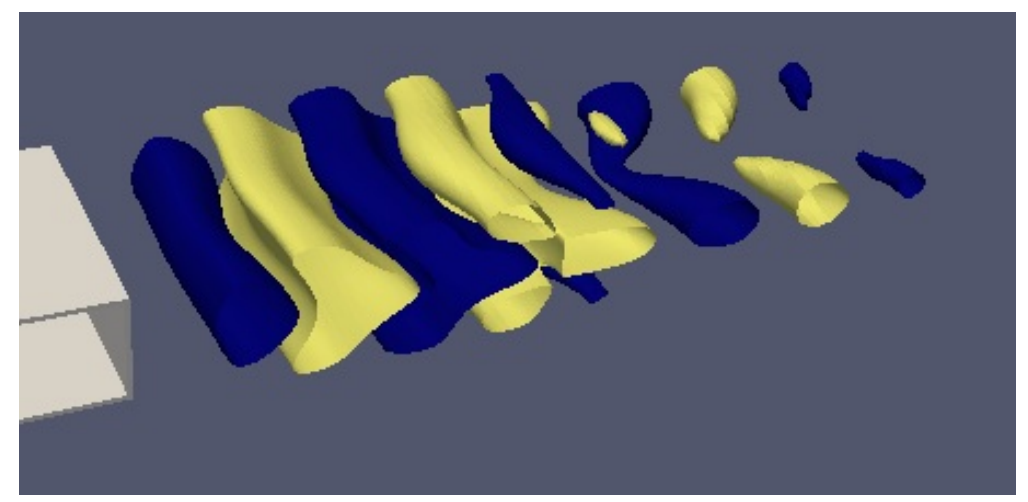

Figure 7. (Color online) The computed TriGlobal eigenmode with suction, $A=0.01$ and $\beta_{B}=1.1$, positive (dark, blue online) and negative (light, yellow online) contours of the vertical disturbance velocity $\hat{v}_{0}(x, y, z)$. The edge of the flat plate, extending from $y=-1$ to $y=1$, is also shown in pale grey.

nor the original eigenmode can indicate which region in the flow has most influence on the eigenvalue change. To know this, we need to overlap the eigenmode correction with the adjoint original eigenmode and the base flow change induced by suction, as explained in $\S 2$, and as demonstrated later in this section.

Now we turn to the comparison between predicted and computed second order eigenvalue changes, and the efficiency of different suction wavelengths $\beta_{B}$ in influencing the stability of the wake flow. The predicted and computed second order eigenvalue changes for all but the shortest wavelength are shown in Fig. 8, demonstrating good qualitative agreement and the same value of the most stabilising wavelength. The shortest wavelength that we tested, $\beta_{B}=30\left(\lambda_{B}=0.2\right)$, is far outside the figure and caused an unobservable change in the eigenvalue for both methods. There are quantitative differences between computed and predicted results which were hard to avoid, since they are computed with two different numerical tools, and the base flow difference had to be interpolated from one grid to another. The absolute difference between prediction and computation is small, less than $10^{-3}$, and therefore easily influenced by numerics. The eigenvalue difference increases quadratically with suction amplitude, and the growth rate decrease is predicted to be $\delta \sigma_{r}=-0.024$ for only $2 \%$ suction amplitude $(A=0.02)$, and $\delta \sigma_{r}=-0.15$ for $5 \%$ suction amplitude $(A=0.05)$. We expect the difference between the two numerical tools to be an absolute difference due to the finite accuracy of solution and interpolation, and hence we expect that the relative difference between prediction and computation will be smaller for even slightly larger suction amplitudes.

The growth rate of the eigenvalue decreases slightly for all cases with suction, in both the prediction and the computation. The eigenvalue is stabilised for a wide range of $\beta_{B}$, approximately $0.4<\beta_{B}<1.6$. The maximal stabilization is reached at $\beta_{B}=1.1$. This corresponds to a wavelength of around 6 . The observed optimal wavelengths for a cylinder flow are longer: Kim \& Choi (2005) obtained 10-12 in our nondimensional coordinates, and Hwang et al. (2013) 12 in our coordinates. The physical reason for the difference between optimal wavelengths in these two configurations could be the confinement. In previous works (Tammisola et al. 2011; Juniper et al. 2011), confinement was shown to alter the base flow as well as the structure of the eigenmode at similar Reynolds numbers. Figure 6 indeed seems shows that the eigenmode correction extends all the way to the surrounding walls, as well as the base flow modification induced by suction does (figure $9 \mathrm{a}-\mathrm{c})$, so they might be affected by confinement. However, the principles behind the 
(a) $\times 10^{-3}$

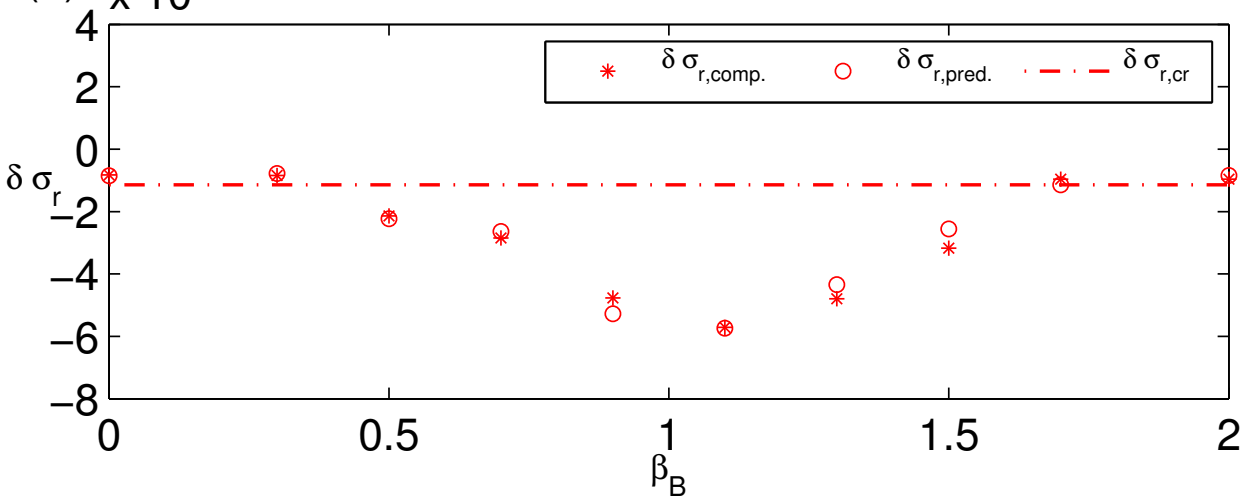

(b) $\times 10^{-3}$

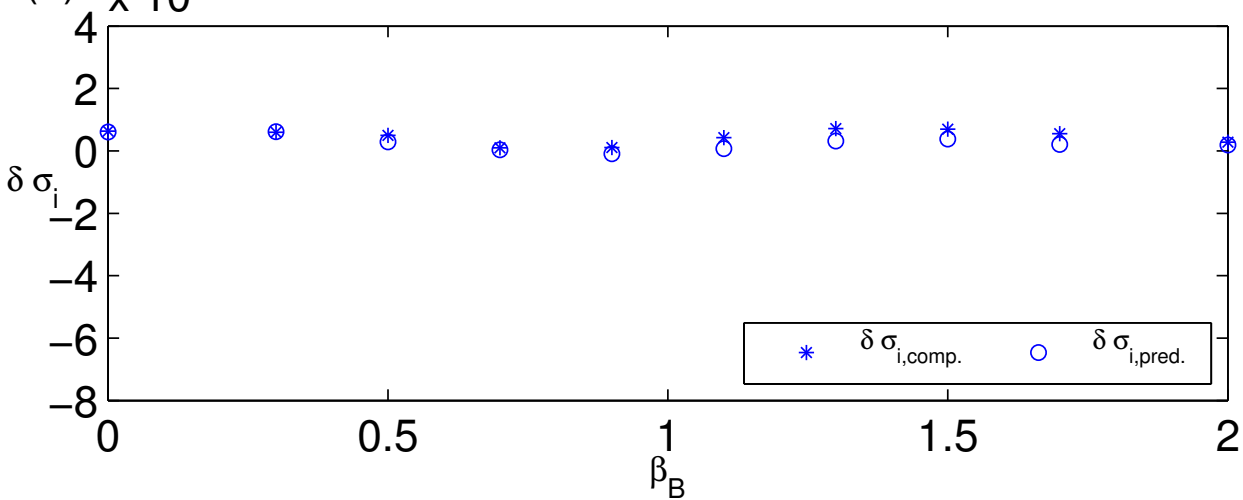

Figure 8. (Color online) The eigenvalue drift for different suction wavenumbers, $\beta_{B}$, for a wake with steady suction where the base flow satisfies the Navier-Stokes equation. Computed and predicted eigenvalue change (see legends): (a) change in the real part, (b) change in the imaginary part. The value where the the growth rate of the suction-modified eigenvalue is zero (neutral stability point) is marked with a dot-dash line in (a).

wavelength selection which we present next are general, and not tied to the specific wake flow in question. First of all, figure 8 shows that the stabilising influence only occurs for relatively long suction wavelengths, and short wavelengths $\left(\beta_{B}>1.7\right)$ have no effect on the eigenvalue. This is consistent with the results in $\S 5.2$, where a fixed base flow modification $\delta \mathbf{U}(x, y)$ was applied with different $\beta_{B}$, and the effect on the eigenvalue decreased with increasing $\beta_{B}$. This also supports the argument presented in $\S 3$ that second order eigenvalue change can occur due to a resonance between unperturbed eigenmodes at $\beta=0$ and $\beta_{B}$, when their eigenvalues are close to each other (for illustration see figure $5)$. The inverse of the difference between the most unstable eigenvalues at $\beta=0$ and $\beta_{B}$ is depicted in figure 10 (f), and explains the general trend that long wavelengths are efficient while short wavelengths are not. However, this argument does not explain why the longest wavelength studied here $\left(\beta_{B}=0.3\right)$ is not stabilizing, and why some slightly shorter wavelengths are performing better. To understand the latter, we consider the structure of the base flow modifications induced by suction.

An important effect was pointed out by Del Guercio et al. (2014): the amplitude of the actual base flow change induced by suction is a function of $\beta_{B}$. In figure $9(\mathrm{a}-\mathrm{c})$, the streamwise velocity difference between suction and no suction base flows is shown 
(a)

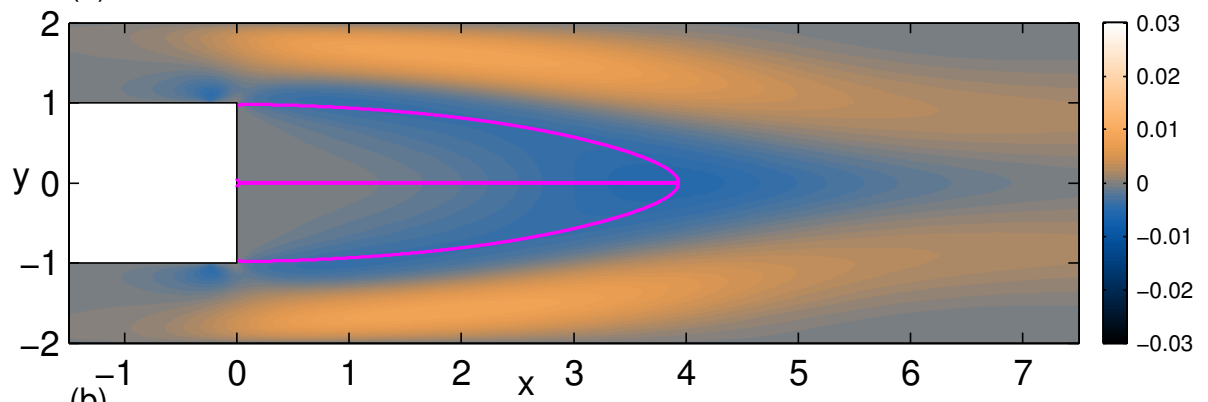
$(\mathrm{b})^{-1}$
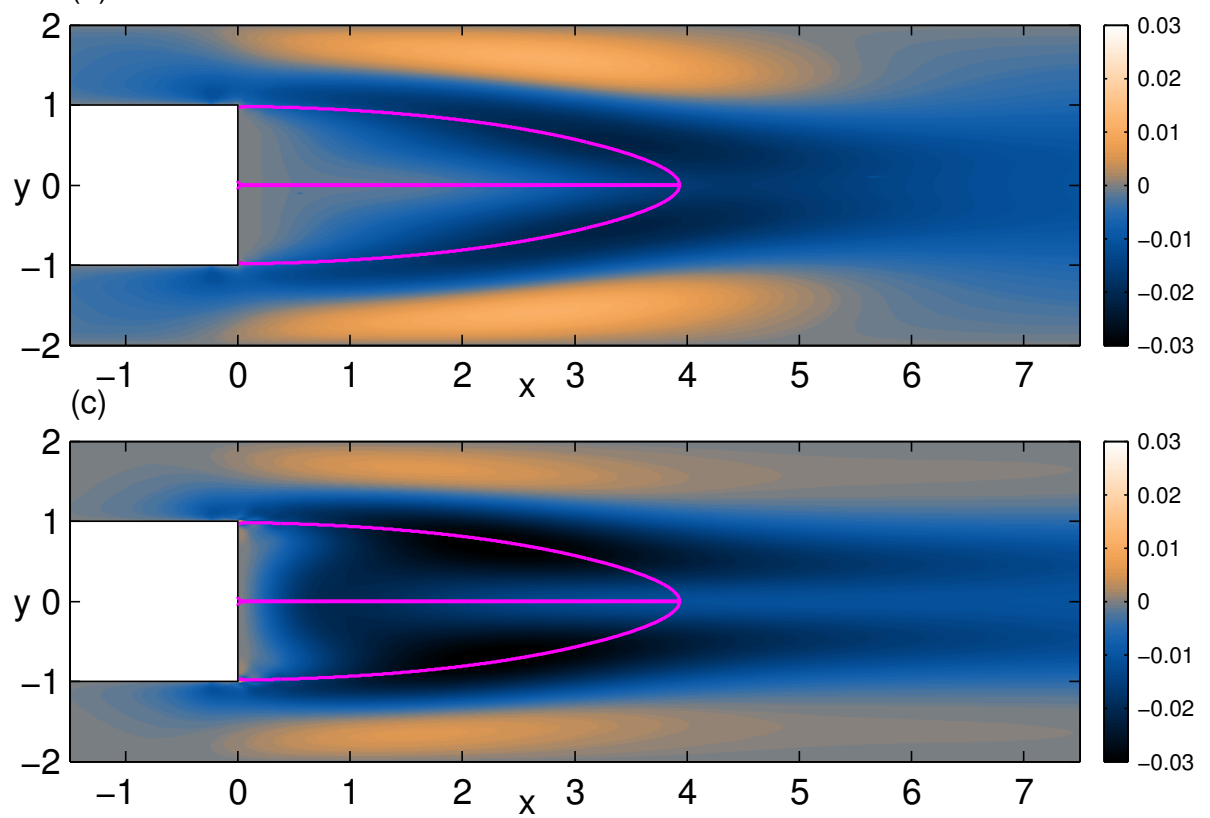

(d)

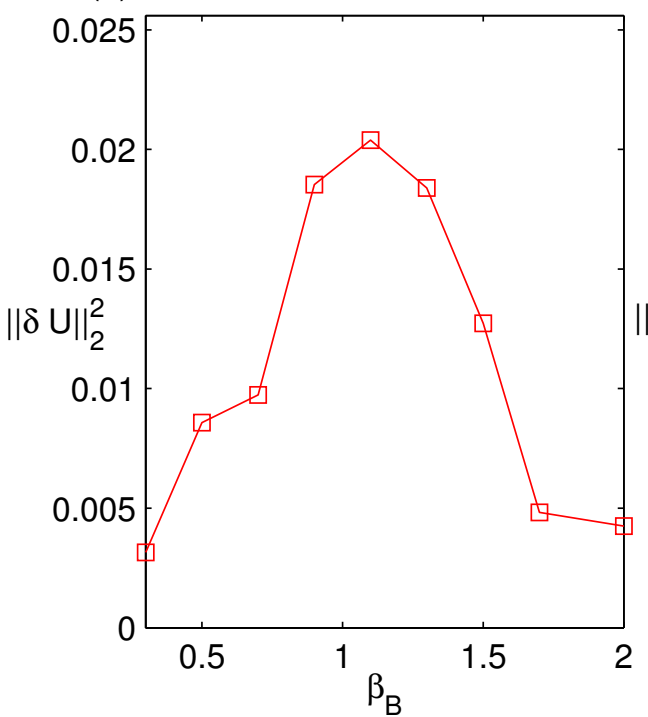

(e) $\times 10^{-3}$

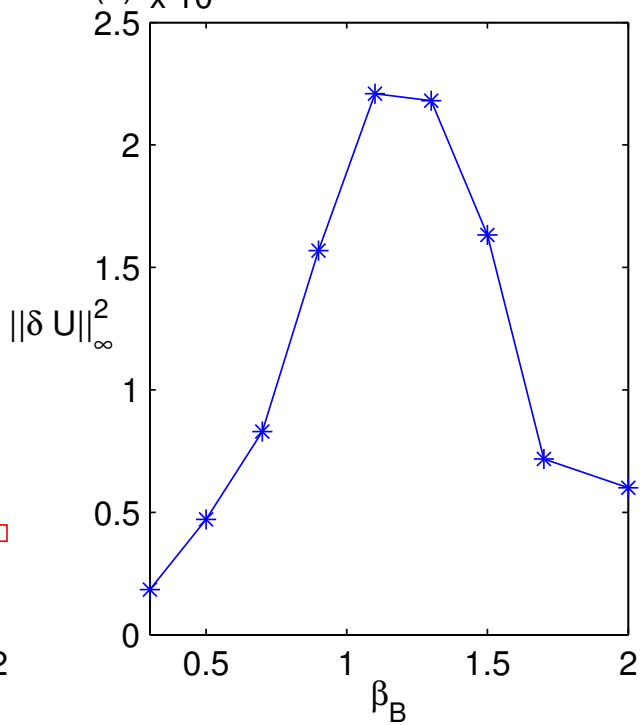

Figure 9. (Color online) The difference between the base flows with and without suction: (a) streamwise velocity difference at $\beta_{B}=0(2 \mathrm{D})$, (b) the streamwise velocity difference at $\beta_{B}=0.5$ at $z=\pi /\left(2 \beta_{B}\right)$, (c) the streamwise velocity difference at $\beta_{B}=2, z=\pi /\left(2 \beta_{B}\right)$, (d) $L_{2}$-norm squared as a function of $\beta_{B}$, and (e) $L_{\infty}$-norm squared as a function of $\beta_{B}$. 
for $\beta_{B}=0$ (a), $\beta_{B}=0.5$ (b), and $\beta_{B}=2$ (c). Two things can be immediately observed. First, spanwise wavy suction $(\mathrm{b}-\mathrm{c})$ is clearly more efficient in modifying the base flow than a spanwise invariant suction (a). Second, the maximal modification is larger for $\beta_{B}=2$ than $\beta_{B}=0.5$. To quantify how efficiently the base flow is modified by suction as a function of $\beta_{B}$, the square of the $L_{2}$-norm and the square of the $L_{\infty}$-norm (maximum norm) of the base flow change are shown for different $\beta_{B}$ in figure 9 (the reason for taking the square is that we expect the eigenvalue drift to be proportional on the square of the base flow change). We can see that both base flow changes are maximal at $\beta_{B}=1.1$. In particular, the square of the $L_{2}$-norm of the base flow change seems to be qualitatively proportional to the growth rate change in figure 8 , confirming that this effect plays an important role in selecting the optimal stabilising wavelength.

It would now be tempting to conclude that the $L_{2}$-norm of the base flow change induced by suction, integrated over the whole domain, determines the stability. The region where eigenvalue changes actually occur (where the base flow modification interacts with the eigenmode correction and the adjoint eigenmode), can be shown by plotting the integrand of $\sigma_{2}$ (Eq. 2.5 or 4.3$)$. In figure $10(\mathrm{a}-\mathrm{d})$, this quantity is shown for different $\beta_{B}$. The eigenvalue is only affected by the base flow changes inside the narrow blue region in these figures, which follows the edge of the recirculation bubble. For all four suction wavelengths, the base flow changes are seen to influence the flow along the outer edge of the recirculation zone for the $2 \mathrm{D}$ base flow (shown in magenta). This effectively coincides with the sensitivity cores shown in $\S 5.2$. The maximum negative value of the integrands (the location in space which contributes to the most stabilizing effect) is in the same location $(x \approx 3.0, y \approx 0.6)$, for all four wavelengths. In figure 10 , we show how the base flow change at the location of maximum sensitivity develops with $\beta_{B}$. This shows the expected trend, with the strongest base flow modification at $\beta_{B}=1.1$.

Our conclusion is that two factors combine to determine the optimal wavelength for stabilization of this flow: (1) The eigenmode resonance occurs at long wavelengths. (2) Medium wavelengths induce largest base flow change inside the sensitivity core region. These competing factors are shown in figures 10 (e) and 10 (f). Regarding how the base flow change due to suction is created, the reader is referred to the recent paper of Del Guercio et al. (2014), where the influence on the cylinder wake base flow is explained to be due to amplification of streaks through the lift-up effect. We expect the same mechanism to be present in this flow, while the base flow changes are slightly modified by confinement.

Finally, it is worth pointing out that the effects described here are based on a global stability analysis, in which the most sensitive region to spanwise wavy perturbations ("sensitivity core") has been identified in the $(x, y)$-plane, and its location related to where the base flow modifications by suction are acting. This was done by mapping the effect of a localized base flow modification in the form of a Dirac delta function. Next, by computing the actual base flow modification induced by suction, and overlapping this with the product of the adjoint eigenmode and eigenmode correction, we found out where the eigenvalue changes due to suction originate. This turned out to coincide well with the sensitivity core. We did not separate the effects of modifying any specific term in Navier-Stokes equations. Our arguments for spanwise wavelength selection are hence slightly different than the ones described in the local analysis of Hwang et al. (2013), detailing the stabilising mechanism of a fixed velocity profile. However, the two analysis are not contradictory, as these two mechanisms might co-exist in a real flow. Del Guercio et al. (2014) point out that spanwise-wavy perturbations introduce a base flow change of a large amplitude through creation of streaks by lift-up effect. We observe that indeed a $2 \mathrm{D}$ suction induces a smaller global base flow modification than a $3 \mathrm{D}$ spanwise wavy 
(a)

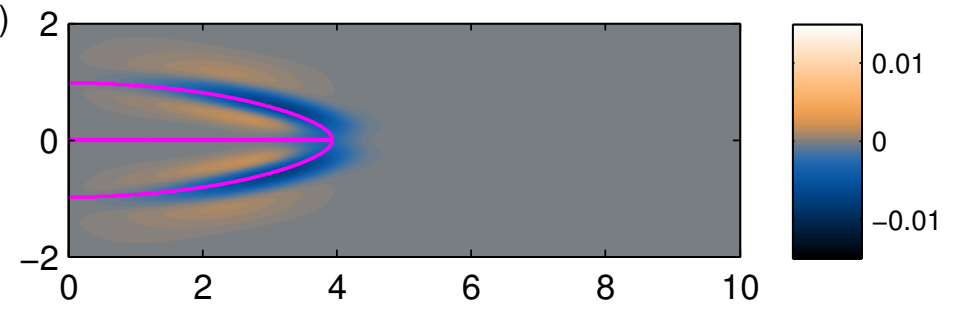

(b)

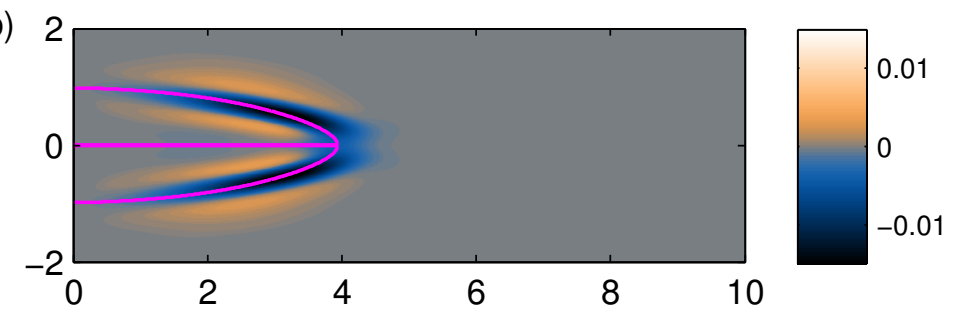

(c)

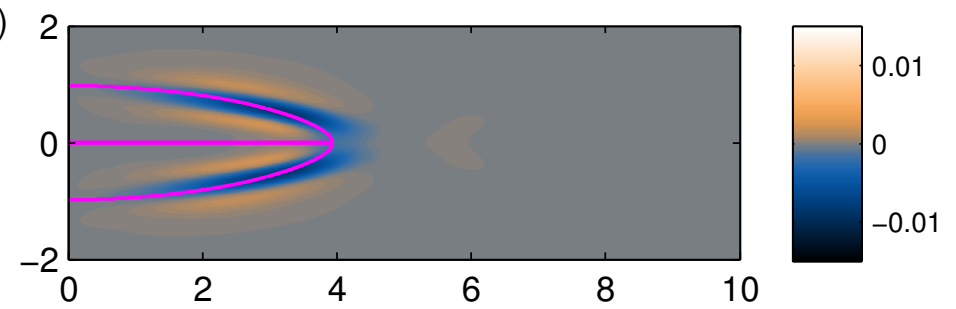

(d)
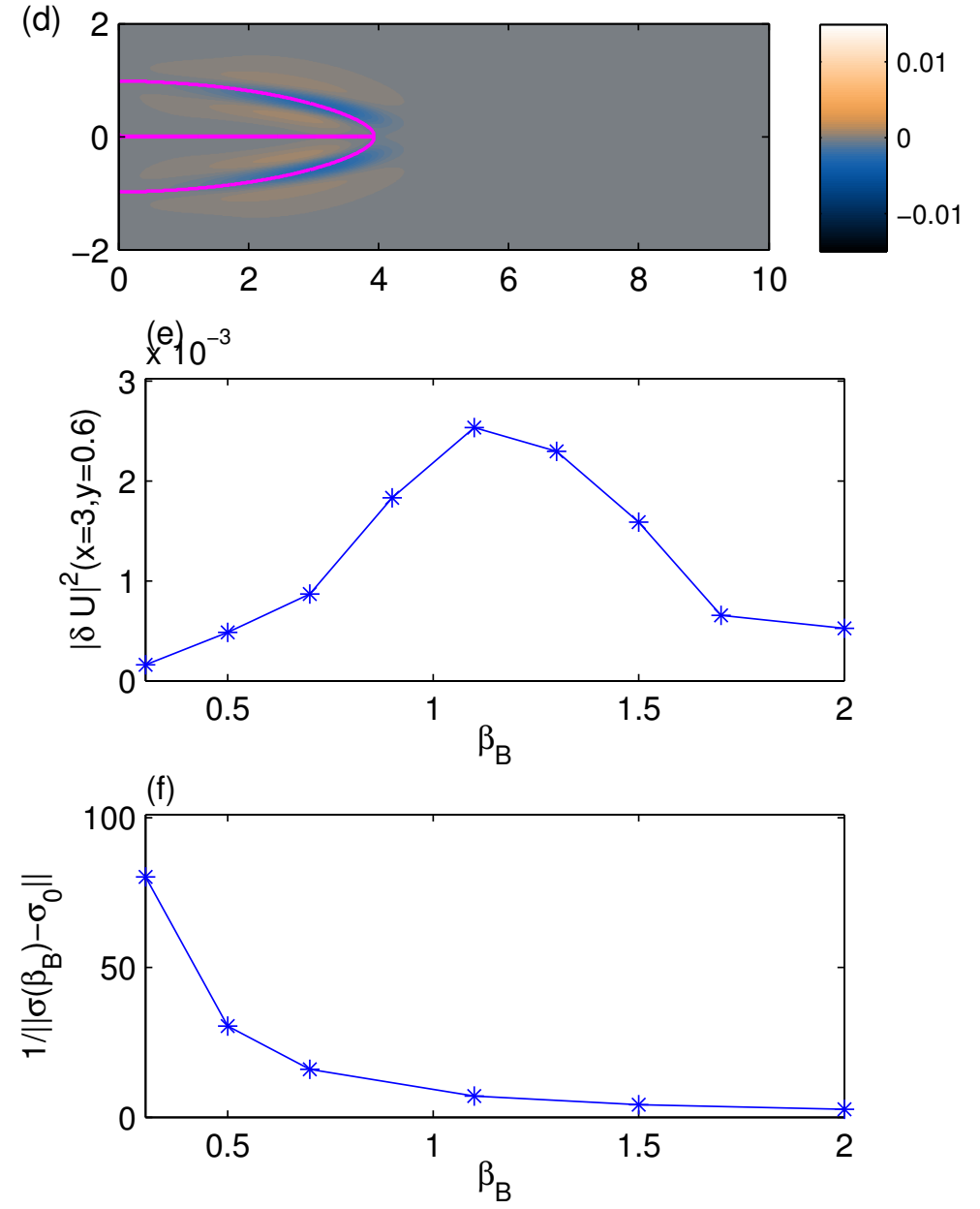

Figure 10. (Color online) (a)-(d): Integrand of the eigenvalue growth rate change (Eq. 2.5). Suction wavelengths: (a) $\beta_{B}=0.7$, (b) $\beta_{B}=1.1$ (middle), (c) $\beta_{B}=1.5$, (d) $\beta_{B}=1.7$. A dark color indicates a stabilizing contribution. The boundary of the recirculation zone of the $2 \mathrm{D}$ base flow is shown by the solid line (magenta online). (e): The amplitude of base flow modification at $x=3.0, y=0.6$, near the maximum negative of the integrands. (f) Inverse of the distance from $\sigma_{0}$ to the closest unperturbed eigenvalue at $\beta=\beta_{B}$. 
suction, in agreement with Del Guercio et al. (2014). However, we extend the previous arguments by identifying the most sensitive region in the $x-y$-plane, and investigating when the base flow modifications induced by suction are inside this most sensitive region.

The vortex-tilting mechanism described in Hwang et al. (2013) might be examined by separating our sensitivities into different components, but this is out of the scope of the present manuscript. The primary aim here is to present and verify theoretical findings applicable to a much wider class of flow problems involving asymmetric perturbations or control.

\section{Conclusions}

The sensitivity of global mode eigenvalues has been generalized by considering the eigenproblem perturbation expansion up to the $2^{\text {nd }}$ order. The theory and numerical solution of the derived bi-global equation system has been used to shed light on how globally unstable flows are influenced by spanwise wavy actuation, for which the standard sensitivity theory predicts no effect. The computational methods presented for computation of the $2^{\text {nd }}$ perturbations are two-dimensional, except for the base flow with suction which was computed in 3D (but could also be computed in 2D as long as the base flow changes linearly with suction amplitude). The two-dimensional predictions were verified by threedimensional computations. The theoretically predicted eigenvalue drift due to spanwise wavy actuation agrees well with the directly computed one. Based on this theory, we argue that the $2^{\text {nd }}$ order effects on the eigenvalue arise from a resonance between the von Karman eigenmode at infinite wavelength (2D) and the same mode at the actuation wavelength. This explains two observations from previous studies: in-phase suction distribution is more efficient than an out-of-phase distribution, and short wavelengths are less efficient than long wavelengths. The optimal wavelength selection is qualitatively explained by showing that the base flow changes induced by suction must be large inside the region most sensitive to spanwise wavy perturbations, called the sensitivity core. From our analysis, this is seen to happen for medium wavelengths. The theory and the eigenmode resonance effect may be relevant to a wide class of problems, for example, the effect of asymmetric inflow profiles on the stability of axisymmetric flows, or flow-acoustics interactions.

\section{Acknowledgment}

This work was supported by the European Research Council through Project ALORS 2590620. This work was performed on the computational facilities provided by the Darwin cluster of the University of Cambridge High Performance Computing Service (http://www.hpc.cam.ac.uk/).

\section{REFERENCES}

Baumgärtel, H. 1984 Perturbation Theory for Matrices and Operators, licensed edition by Birkhäuser Verlag edn. Akademie Verlag Berlin.

DAVIS, T. A. 2004 Algorithm 832: Umfpack, an unsymmetric-pattern multifrontal method. Transactions on Mathematical Software 30 (2), 196-199.

Del Guercio, G., Cossu, C. \& Pujals, G. 2014 Stabilizing effect of optimally amplified streaks in parallel wakes. Journal of Fluid Mechanics 739, 37-56.

Fischer, P. F. 1997 An Overlapping Schwarz Method for Spectral Element Solution of the Incompressible Navier-Stokes Equations. Journal of Computational Physics 133, 84-101. 
Giannetti, F. \& Luchini, P. 2007 Structural sensitivity of the first instability of the cylinder wake. Journal of Fluid Mechanics 581, 167-197.

Hinch, E. J. 1991 Perturbation Methods. Cambridge: Cambridge University Press.

Hwang, Y., Kim, J. \& ChOI, H. 2013 Stabilization of absolute instability in spanwise wavy two-dimensional wakes. Journal of Fluid Mechanics 727, 346-378.

Juniper, M., Tammisola, O. \& Lundell, F. 2011 The local and global stability of confined planar wakes at intermediate reynolds number. Journal of Fluid Mechanics 686, 218-238.

Kiм \& Chor 2005 Distributed forcing of flow over a circular cylinder. Physics of Fluids 17, 033103.

Kolmogorov, A. N. 1954 On the conservation of conditionally periodic motions under small perturbation of the hamiltonian. Dokl. Akad. Nauk SSR 74, 527-530.

Lashgari, I., Tammisola, O., Brandt, L. \& Juniper, M. P. 2013 The planar X-junction flow: Stability and control. Submitted to Journal of Fluid Mechanics .

LehoucQ, R. B., Sorensen, D. C. \& YANG, C. 1998 ARPACK users' guide : solution of largescale eigenvalue problems with implicitly restarted Arnoldi methods. Philadelphia: SIAM.

Lieu, B. K., Moarref, R. \& Jovanovic, M. R. 2010 Controlling the onset of turbulence by streamwise travelling waves. Part 2. Direct numerical simulation. Journal of Fluid Mechanics 663, 100-119.

Luchini, P. \& Bottaro, A. 2014 Adjoint equations in stability analysis. Annual Review of Fluid Mechanics 46, 493-517.

Marquet, O., Sipp, D. \& JaCquin, L. 2008 Sensitivity analysis and passive control of cylinder flow. Journal of Fluid Mechanics 615, 221-252.

Maschioff, K. J. \& Sorensen, D. C. 1996 P_ARPACK: An efficient portable large scale eigenvalue package for distributed memory parallel architectures. In Proceedings of the Third International Workshop on Applied Parallel Computing, Industrial Computation and Optimization, pp. 478-486. London, UK: Springer-Verlag.

MoArref, R. \& Jovanovic, M. R. 2010 Controlling the onset of turbulence by streamwise travelling waves. Part 1. Receptivity analysis. Journal of Fluid Mechanics 663, 70-99.

Pralits, J. O., Byström, M., Hanifi, A. \& Henningson, D. S. 2007 Optimal disturbances in three-dimensional boundary-layer flows. Ercoftac Bulletin 74, 23-31.

Tammisola, O., Lundell, F., Schlatter, P., Wehrfritz, A. \& Söderberg, L. D. 2011 Global linear and nonlinear stability of viscous confined plane wakes with co-flow. Journal of Fluid Mechanics 675, 397-434.

Theofilis, V. 2003 Advances in global linear instability analysis of nonparallel and threedimensional flows. Progress in Aerospace Sciences 39, 249-315. 